\title{
Pseudo-Inverse Linear Discriminants for the Improvement of Overall
}

\section{Classification Accuracies}

\author{
Gao Daqi, Dastagir Ahmed, Guo LIli, Wang Zejian, Wang Zhe \\ Department of Computer Science, State Key Laboratory of Bioreactor Engineering, East China University of Science and Technology, \\ Shanghai 200237, China
}

(C) 2016. This manuscript version is made available under the Elsevier user license http://www.elsevier.com/open-access/userlicense/1.0/ 


\section{Abstract:}

This paper studies the learning and generalization performances of pseudo-inverse linear discriminant (PILDs) based on the processing minimum sum-of-squared error $\left(\mathrm{MS}^{2} \mathrm{E}\right)$ and the targeting overall classification accuracy (OCA) criterion functions. There is little practicable significance to prove the equivalency between a PILD with the desired outputs in reverse proportion to the number of class samples and an FLD with the totally projected mean thresholds. When the desired outputs of each class are assigned a fixed value, a PILD is partly equal to an FLD. With the customarily desired outputs $\{1,-1\}$, a practicable threshold is acquired, which is only related to sample sizes. If the desired outputs of each sample are changeable, a PILD has nothing in common with an FLD. The optimal threshold may thus be singled out from multiple empirical ones related to sizes and distributed regions. Depending upon the processing $\mathrm{MS}^{2} \mathrm{E}$ criterions and the actually algebraic distances, an iterative learning strategy of PILD is proposed, the outstanding advantages of which are with limited epoch, without learning rate and divergent risk. Enormous experimental results for the benchmark datasets have verified that the iterative PILDs with optimal thresholds have good learning and generalization performances, and even reach the top OCAs for some datasets among the existing classifiers.

Keywords: Pseudo-inverse linear discriminants (PILDs); Fisher linear discriminants (FLDs); Threshold optimization; Iterative learning; Overall classification accuracies. 


\section{Introduction}

Performances of classifiers can be evaluated from two aspects: processing and targeting criterions. The most often-used processing evaluation criterion functions are the minimum sum-of-squared errors (MS $\left.{ }^{2} E s\right)$ and the least-mean-squared (LMS) errors (Duda, Hart, \& Stork, 2000). And the most conventional targeting evaluation criterion functions are the overall classification accuracies (OCAs), or called the overall recognition rates (ORRs) or the overall error rates (OERs) (Huang \& Ling, 2005). There are two main learning procedures: analytical and iterative, to determine the parameters of classifiers (Duda, Hart, \& Stork, 2000; Suykens et al., 2002). Specified to a linear classifier $\pi$ : $\theta+\boldsymbol{w}^{T} \boldsymbol{X}=0$, two main types of algorithms to determine the thresholds $\theta$ and the weight vectors $\boldsymbol{w}$ are as follows: $(A)$ analytical procedures, e.g., the $\mathrm{MS}^{2} \mathrm{E}$ solutions, depending upon the processing $\mathrm{MS}^{2} \mathrm{E}$ criterion functions $J(\theta, \boldsymbol{w})=\|\theta \mathbf{1}+\boldsymbol{X} \mathbf{w}-\boldsymbol{d}\|^{2}$ by one-time calculation of explicit equations in a lump, and $(B)$ iterative procedures, e.g., the gradient descent solutions, mainly depending upon the processing LMS error functions $J(\theta(\tau), \boldsymbol{w}(\tau))=\sum_{p}\left(\theta(\tau)+\boldsymbol{w}^{T}(\tau) \boldsymbol{x}_{p}-d_{p}\right)^{2}$ by numerous repeated iterations (Duda, Hart, \& Stork, 2000; Koford \& Groner, 1966). The analytical procedures have two advantages over the iterative ones: (i) fast computational speeds and (ii) without local minimums.

Fisher linear discriminants (FLDs), also called linear discriminant analyses (LDAs), are very popular (Koford \& Groner, 1966; Billings \& Lee, 2002; Cawley \& Talbot, 2003). Following the force of habit, FLDs are often taken for and in particular equated with linear classifiers (Raudys \& Duin, 1998; Cooke, 2002; Rozza et al., 2012). However, it must be clarified that FLDs are only a type of analytical learning algorithms based on the Rayleigh quotients $J(\boldsymbol{w})=\frac{\boldsymbol{w}^{T} \boldsymbol{S}_{B} \boldsymbol{w}}{\boldsymbol{w}^{T} \boldsymbol{S}_{W} \boldsymbol{w}}$ in the Bayesian decision theory (Duda, Hart, \& Stork, 2000). Here, $\boldsymbol{S}_{B}$ and $\boldsymbol{S}_{W}$ are the betweenand within-class scatter matrices, respectively. Of course, FLDs are more used as a kind of feature extraction tools than a type of classifiers, especially in the image processing fields, e.g., the classical Fisherfaces (Belhumeur, Hespanha, \& Kriegman, 2007). Single-layer perceptrons are another type of linear classifiers (Suykens et al., 2002; Elozondo, 2006; Escalera et al., 2008). They mainly employ the iterative learning procedures, e.g., back-propagation, to determine the thresholds $\theta$ and the weights $\boldsymbol{w}$.

Pseudo-inverse linear discriminants (PILDs), another kind of analytical learning algorithm for linear classifiers, obtain the $\theta$ and $\boldsymbol{w}$ according to the processing $\mathrm{MS}^{2} \mathrm{E}$ criterion functions (Duda, Hart, \& Stork, 2000). Note that the word "pseudo-inverse" here is borrowed to show that the equation for calculating the $\theta$ and $\boldsymbol{w}$ formally contains the Moore-Penrose inverse item (Raudys \& Duin, 1998; Hoyle, 2011; Tapson \& Schaik, 2013), but not to imply the non-invertibility of a rectangular matrix. For the purpose of simplicity, sometimes the abbrev terms "PILDs" are inexactly used to imply "linear classifiers with pseudo-inverse discriminant algorithm", and so are "FLDs" to "linear classifiers with Fisher discriminant algorithm", henceforth.

A PILD has a bit higher computational complexity than an FLD, because the former processes an $(m+1) \times(m+1)$ square matrix while the latter does an $m \times m$ one. However, a PILD has two main advantages over an FLD: $(A)$ the $\theta$ and the $\boldsymbol{w}$ are simultaneously obtained by solving a single analytical equation, and $(B)$ the $\mathrm{MS}^{2} \mathrm{E}$ criterion function could be further developed to optimize the $\theta$ and $\boldsymbol{w}$. It has been proved that a PILD with the specially desired outputs in reverse proportion to the number of samples is equivalent to an FLD with the totally projected mean (TPM) threshold (Duda, Hart, \& Stork, 2000). FLDs are quite popular (Billings \& Lee, 2002; Raudys \& Duin, 1998; Cooke, 2002); however, PILDs are rarely applied to practice. The true reason lies in the fact that PILDs are not ideal in OCAs.

Real-world datasets are of diverse sizes and distributed regions (Galar et al., 2012; Nicolas, Javier, \& Aida de, 2012). Class imbalance is an objective being (Huang \& Ling, 2005; Fernandez et al., 2008; Cano, Zafra, \& Ventura, 2013). Taking a binary-class problem $\left\{\omega_{1}, \omega_{2}\right\}$ for example, there are the following four possible cases: $(A) \omega_{1}$ is equal to $\omega_{2}$ both in size and in region; $(B) \omega_{1}$ is larger in size but smaller than or equal to $\omega_{2}$ in region; $(C) \omega_{1}$ is larger in region but smaller than or equal to $\omega_{2}$ in size; $(D) \omega_{1}$ is larger both in size and in region than $\omega_{2}$. It is difficult for a single $\theta$ and even a single $\boldsymbol{w}$ obtained by using an analytical equation in one-lump computation to suit all cases.

In order to solve the classification problems of imbalanced datasets, we can proceed from the following approaches: ( $i$ ) data and (ii) classifiers as well as algorithms. In the data levels are diversely over-, under-, and synthetic-sampling techniques (Nicolas, Javier, \& Aida de, 2012; Barua et al., 2014; Galar et al., 2013). Decision trees (Wang \& Yao, 2013), support vector machines (SVMs) (Tang et al., 2009; Maldonado \& Lopez, 2014), neural networks (Castro \& Braga, 2013), FLDs (Rozza et al., 2012; Gao, Ding, \& Zhu, 2014) and even $k$-nearest- neighbor 
rules (Barua et al., 2014) are the main classification models. And different cost-sensitive learning algorithms, e.g., bagging and boosting (Nicolas, Javier, \& Aida de, 2012; Shen \& Li, 2010; Hand \& Till, 2001) as well as some criterion functions, especially, the areas under the ROC curves (AUCs) (Huang \& Ling, 2005; Bradley, 1997; Adams $\&$ Hand, 1999), are paid much attention. Comparatively speaking, PILDs do not attract enough concern.

This paper aims at improving the learning and generalization performances of PILDs based on the processing $\mathrm{MS}^{2} \mathrm{E}$ and the targeting OCA criterion functions (Bradley, 1997; Adams \& Hand, 1999), and will devote to addressing the following problems:

(a) Is a PILD with the designated outputs in reverse proportion to the number of samples indeed equal to an FLD with the TPM threshold? What if the desired outputs are taken the conventional values $d_{p} \in\{1,-1\}$ (Duda, Hart, \& Stork, 2000; Koford \& Groner, 1966; Rozza et al., 2012)?

(b) Are the weight vectors and the thresholds calculated by the pseudo-inverse solution optimal? If not, how to optimize them in a comprehensive consideration of sample sizes and distributed regions (Gao, Ding, \& Zhu, 2014)?

(c) Is it reasonable to allocate the desired outputs of each class a fixed value? If not, how to re-allocate the rational ones for all training samples, including both the correct and the misclassified ones (Duda, Hart, \& Stork, 2000; Adams \& Hand, 1999)?

(d) How to introduce an iterative learning strategy to the analytical PILDs in order to further optimize the thresholds and weights (Duda, Hart, \& Stork, 2000; Gao, Ding, \& Zhu, 2014)?

Motivated by the troublesome issues above, this paper concentrates attention on improving the learning and generalization of PILDs from the aspects of thresholds, weight vectors and data in the algorithm level. The contributions of this work are summarized in the following:

(A) A PILD is not exactly equivalent to an FLD even if the desired outputs in the PILD are in reverse proportion to the number of samples. Several examples are given to support the argument.

(B) A PILD is partly equivalent to an FLD when all training samples of each class are allocated a fixedly desired output. And they two have nothing in common when all the training samples are assigned with changeably desired outputs.

(C) The most often-used TPM thresholds usually behave poor in OCAs because of the unsuitable truncation (Gao, Ding, \& Zhu, 2014). A simple practicable $\mathrm{MS}^{2} \mathrm{E}$ threshold comes into being by using the customarily desired outputs $d_{p} \in\{1,-1\}$. Furthermore, a rational threshold may be obtained by using the actually algebraic distances as the desired outputs.

(D) Multiple empirical thresholds are developed in a comprehensive consideration of sizes and regions. The optimal thresholds are singled out from among them aiming at the best OCAs.

(E) The iterative learning strategy of PILDs is proposed by means of the processing $\mathrm{MS}^{2} \mathrm{E}$ criterion and the actually 
algebraic distances, which is with limited epochs, without learning rate and divergent risk.

We stress that this work is the development of our earlier work (Gao, Ding, \& Zhu, 2014); therefore we will always pay much attention on the difference between them two. The rest of this paper is organized as follows: Section 2 introduces the related work of PILDs. In Section 3, the relationship between PILDs and FLDs is clarified, and three examples are given to verify their similar and different places. Section 4 develops a series of empirical thresholds related to sizes and regions. Section 5 goes into details on the iterative learning strategy of PILDs based on the processing $\mathrm{MS}^{2} \mathrm{E}$ criterion functions and the changeably desired outputs. Section 6 presents numerous experimental results for the real-world benchmark datasets to demonstrate the superior learning and generalization performances of PILDs. Finally, we will conclude this work in Section 7.

\section{Related Work}

The augmented vector of a sample $\boldsymbol{x}_{p} \in R^{m}$ is written as $\tilde{\boldsymbol{X}}_{p}=\left(1, \boldsymbol{x}_{p}\right)^{T} \in R^{m+1}$, where $m$ is the number of dimensions in the input space. The augmented matrix of a dataset $\boldsymbol{X}=\left(\boldsymbol{x}_{1}, \ldots, \boldsymbol{X}_{N}\right)^{T} \in R^{N \rtimes m}$ is marked as $\tilde{\boldsymbol{X}}=(\mathbf{1}, \boldsymbol{X}) \in R^{N \times(m+1)}$, where $N$ is the number of samples. Let $d_{p}$ be the desired output for $\boldsymbol{x}_{p}$, the desired output vector for all the $N$ samples is $\boldsymbol{d}=\left(d_{1}, \ldots, d_{N}\right)^{T} \in R^{N}$. For a linear classifier $\pi: \theta+\boldsymbol{W}^{T} \boldsymbol{X}=0$, the pseudo-inverse learning algorithm minimizes the sum of squared error:

$$
J(\theta, \boldsymbol{w})=\left\|\tilde{\boldsymbol{X}}\left(\begin{array}{c}
\theta \\
\boldsymbol{w}
\end{array}\right)-\boldsymbol{d}\right\| \|^{2}=\sum_{p=1}^{N}\left(\theta+\boldsymbol{w}^{T} \boldsymbol{x}_{p}-d_{p}\right)^{2}
$$

Equation (1) is also called the processing $\mathrm{MS}^{2} \mathrm{E}$ evaluation function. If the $(m+1)$-by-(m+1) square matrix $\tilde{\boldsymbol{X}}^{T} \tilde{\boldsymbol{X}}$ is invertible, the threshold $\theta$ and the weight vector $\boldsymbol{W} \in R^{m}$ can be uniquely determined by the $\mathrm{MS}^{2} \mathrm{E}$ solution of Eq. (1):

$$
(\theta, \boldsymbol{w})^{T}=\left(\tilde{\boldsymbol{X}}^{T} \tilde{\boldsymbol{X}}\right)^{-1} \tilde{\boldsymbol{X}}^{T} \boldsymbol{d}
$$

Here, $\left(\tilde{\boldsymbol{X}}^{T} \tilde{\boldsymbol{X}}\right)^{-1} \tilde{\boldsymbol{X}}^{T}$ is called the pseudo-inverse of $\tilde{\boldsymbol{X}}$. In fact, Eq. (2) is attained by letting $\left.\partial J(\theta, \boldsymbol{w}) / \partial \theta, \boldsymbol{w}\right)=\boldsymbol{0}$. If singular, $\tilde{\boldsymbol{X}}^{T} \tilde{\boldsymbol{X}}$ is often added a tiny perturbation $\delta \boldsymbol{l}$, where $\boldsymbol{I}$ is an $(m+1) \times(m+1)$ identity matrix and $\delta$ is a perturbation constant. At the moment, the pseudo-inverse solution of Eq. (2) becomes $(\theta, \boldsymbol{w})^{T}=\left(\tilde{\boldsymbol{X}}^{T} \tilde{\boldsymbol{X}}+\delta \boldsymbol{l}\right)^{-1} \tilde{\boldsymbol{X}}^{T} \boldsymbol{d}$.

For a binary-class dataset $\boldsymbol{X}=\left\{\boldsymbol{X}^{11}, \boldsymbol{X}^{(2\}}\right\}$, where $\boldsymbol{X}^{(1\}} \in R^{N 1 \times m}$ and $\boldsymbol{X}^{22\}} \in R^{N 2 \times m}, N_{1}$ and $N_{2}$ are the numbers of samples in the two classes $\omega_{1}$ and $\omega_{2}$, respectively, and $N=N_{1}+N_{2}$, Duda, Hart, \& Stork (2000) had pointed out that the condition of equivalence of a PILD to an FLD is to let the desired output vector be

$$
\boldsymbol{d}=\left(\frac{N}{N_{1}} \mathbf{1}_{1},-\frac{N}{N_{2}} \mathbf{1}_{2}\right)^{T}
$$

and the resulting $\theta$ and $\boldsymbol{w}$ are

$$
\begin{aligned}
& \theta=-\boldsymbol{w}^{T} \boldsymbol{\mu}=-\boldsymbol{w}^{T}\left(\frac{N_{1} \boldsymbol{\mu}_{1}+N_{2} \boldsymbol{\mu}_{2}}{N}\right)=-\mu_{\boldsymbol{w}}=\theta_{1} \\
& \boldsymbol{w}=\alpha N \boldsymbol{S}_{W}^{-1}\left(\boldsymbol{\mu}_{1}-\boldsymbol{\mu}_{2}\right) \propto \boldsymbol{S}_{W}^{-1}\left(\boldsymbol{\mu}_{1}-\boldsymbol{\mu}_{2}\right)
\end{aligned}
$$

Here, $\alpha$ is an unimportant scalar factor, $\boldsymbol{\mu}_{1}$ and $\boldsymbol{\mu}_{2}$ are two class-mean vectors, $\boldsymbol{\mu}$ is the total mean vector, and $\mu_{\boldsymbol{w}}=\boldsymbol{w}^{T} \boldsymbol{\mu}$ is the totally projected mean in the direction $\boldsymbol{w}$. Equation (4) is a widely used threshold formula in FLDs (Duda, Hart, \& Stork, 2000; Billings \& Lee, 2002; Cawley \& Talbot, 2003). $\theta_{1}$ is in essence the negative TPM, i.e., $-\mu_{w}$, in the direction $\boldsymbol{w}$, called the TPM threshold, and only related to sample sizes but not to distributed regions. Let $N_{1}$ is far larger than $N_{2}$, namely $N_{1}>>N_{2}$, we have $\theta_{1}=-\mu_{w} \approx \mu_{1}$, which is quite disadvantageous to the majority classes and thus detrimental to the OCAs (Gao, Ding, \& Zhu, 2014). The within-class scatter matrix $\boldsymbol{S}_{W} \in R^{m \times m}$ in $\left\{\omega_{1}, \omega_{2}\right\}$ is calculated by

$$
\boldsymbol{S}_{W}=\boldsymbol{S}_{1}+\boldsymbol{S}_{2}=\sum_{j=1}^{2} \sum_{\boldsymbol{x}_{p} \in \omega_{j}}\left(\boldsymbol{x}_{p}-\boldsymbol{\mu}_{j}\right)\left(\boldsymbol{x}_{p}-\boldsymbol{\mu}_{j}\right)^{T}
$$

There exist the following serious defects in Eq. (3): (A) It is not reasonable to assign all training samples of each class with the same desired output values. If so, it implies that all the training samples in the class are located on the 
same hyperplane, which apparently conflicts with the facts. $(B)$ The class imbalance of distributed regions is not taken into account at all. In other words, both $N / N_{1}$ and $-N / N_{2}$ are only related to sample sizes. $(C)$ The way of designating the desired outputs $\left\{N / N_{1},-N / N_{2}\right\}$ is not only inconsistent with the conventional form but also unpractical. For instance, supposing $N_{1}=5$ and $N_{2}=1$, the desired outputs for 6 samples will be $d_{1}=\ldots=d_{5}=6 / 5=1.2$ and $d_{6}=-6 / 1=-6$, a group of absurd values. Based on the above three views, we can infer with certainty that the TPM threshold $\theta_{1}=-\mu_{w}$ will behave poor in OCAs for the datasets with serious imbalance of distributed regions. In the next section, we will make clear that the $\theta_{1}$ is only the approximate expression of a reasonable threshold by the $\mathrm{MS}^{2} \mathrm{E}$ criterion.

It is easy to make decision for a binary-class problem. For a multi-class problem, say an $n$-class problem, an effective approach is first to decompose it into $n(n-1) / 2$ two-class problems and then solve them by using $n(n-1) / 2$ classifiers, one for each. At the moment, the classical majority vote rule is employed to make decision (Suykens et al., 2002; Liu, Hao, \& Tsang, 2008). If not declared in particular, we employ that kind of decision rule hereafter.

\section{Relation to Fisher Linear Discriminant}

In this section, we will answer the following two questions: (A) Is a PILD indeed equivalent to an FLD even if the desired outputs are allocated to be $d_{p} \in\left\{N / N_{1},-N / N_{2}\right\}$ for all $p$ ? (B) What if $d_{p} \in\{1,-1\}$ ?

For the purpose of comparison, we directly give another widely-used threshold in FLDs (Suykens et al., 2002; Koford \& Groner, 1966; Tang et al., 2009; Hoyle, 2011):

$$
\theta=-\boldsymbol{w}^{T}\left(\frac{\boldsymbol{\mu}_{1}+\boldsymbol{\mu}_{2}}{2}\right)=-\frac{\mu_{w}^{(1)}+\mu_{w}^{(2)}}{2}=\theta_{2}
$$

$\theta_{2}$ is actually the midpoint of two projected means in the direction $\boldsymbol{w}$, called the midpoint-of-projected-means (MPM) threshold. As a matter of fact, an SVM also determines the bias in a similar consideration in order to make the separating hyperplane to be just located in the middle of two parallel hyperplanes formed by the support vectors (Suykens et al., 2002; Elozondo, 2006). If $N_{1}=N_{2}$, then $\theta_{2}=\theta_{1}$. Therefore, Eq. (7) can be looked upon as a specific case of Eq. (4).

\subsection{Three Synthetic Examples}

Suppose there are three synthetic binary-class datasets in a 2-dimensional space. The sample distributions are as follows:

Dataset 1 , class $\omega_{1}:\left\{(\alpha, 0)^{T} ;(0,1)^{T} ;(0,-1)^{T}\right\}$; class $\omega_{2}:\left\{(1,0)^{T}\right\} . N_{1}=3, N_{2}=1$, and $N=4$.

Dataset 2, class $\omega_{1}:\left\{(\alpha, \alpha)^{T} ;(0,1)^{T} ;(1,0)^{T}\right\}$; class $\omega_{2}:\left\{(1,1)^{T}\right\} . N_{1}=3, N_{2}=1$, and $N=4$.

Dataset 3, class $\omega_{1}:\left\{(1,2)^{T},(2,0)^{T}\right\}$; class $\omega_{2}:\left\{(3,1)^{T},(2,3)^{T}\right\} . N_{1}=N_{2}=2$, and $N=4$.

Design PILDs with $d_{p} \in\left\{N / N_{1},-N / N_{2}\right\}$ or $d_{p} \in\{1,-1\}$ and FLDs with $\theta_{1}$ or $\theta_{2}$ to solve them, respectively. In particular, for Datasets 1 and 2, determine the active regions of the value $\alpha$.

For Dataset $1, \quad \tilde{\boldsymbol{X}}^{T} \tilde{\boldsymbol{X}}=\left(\begin{array}{ccc}4 & \alpha+1 & 0 \\ \alpha+1 & \alpha^{2}+1 & 0 \\ 0 & 0 & 2\end{array}\right) \quad, \quad$ the $\operatorname{determinant} \operatorname{det}\left(\tilde{\boldsymbol{X}}^{T} \tilde{\boldsymbol{X}}\right)=6 \alpha^{2}-4 \alpha+6>0$; $\boldsymbol{S}_{W}=\left(\begin{array}{cc}\frac{2}{3} \alpha^{2} & 0 \\ 0 & 2\end{array}\right)$, and $\operatorname{det}\left(\boldsymbol{S}_{W}\right)=\frac{4}{3} \alpha^{2} \geq 0$, and the investable condition of $\boldsymbol{S}_{W}$ is $\alpha \neq 0$.

For Dataset 2, $\quad \tilde{\boldsymbol{X}}^{T} \tilde{\boldsymbol{X}}=\left(\begin{array}{ccc}4 & \alpha+2 & \alpha+2 \\ \alpha+2 & \alpha^{2}+2 & \alpha^{2}+1 \\ \alpha+2 & \alpha^{2}+1 & \alpha^{2}+2\end{array}\right), \quad$ the determinant $\operatorname{det}\left(\tilde{\boldsymbol{X}}^{T} \tilde{\boldsymbol{X}}\right)=6 \alpha^{2}-8 \alpha+4>0$; $\boldsymbol{S}_{W}=\frac{1}{3}\left(\begin{array}{cc}2 \alpha^{2}-2 \alpha+2 & 2 \alpha^{2}-2 \alpha-1 \\ 2 \alpha^{2}-2 \alpha-1 & 2 \alpha^{2}-2 \alpha+2\end{array}\right), \operatorname{det}\left(\boldsymbol{S}_{W}\right)=\frac{1}{3}(2 \alpha-1)^{2} \geq 0$, and the investable condition of $\boldsymbol{S}_{W}$ is $\alpha \neq 1 / 2$.

Dataset 3 is just Example 1 in Subsection 5.8.1 in the textbook by Duda, Hart, \& Stork (2000). For the dataset, we 
have $\quad \tilde{\boldsymbol{X}}^{T} \tilde{\boldsymbol{X}}=\left(\begin{array}{ccc}4 & 8 & 6 \\ 8 & 18 & 11 \\ 6 & 11 & 14\end{array}\right) \quad, \quad\left(\tilde{\boldsymbol{X}}^{T} \tilde{\boldsymbol{X}}\right)^{-1}=\frac{1}{36}\left(\begin{array}{ccc}131 & -46 & -20 \\ -46 & 20 & 4 \\ -20 & 4 & 8\end{array}\right) \quad, \quad \boldsymbol{S}_{1}=\boldsymbol{S}_{2}=\left(\begin{array}{cc}1 / 2 & -1 \\ -1 & 2\end{array}\right) \quad, \quad$ and $\boldsymbol{S}_{W}=\boldsymbol{S}_{1}+\boldsymbol{S}_{2}=\left(\begin{array}{cc}1 & -2 \\ -2 & 4\end{array}\right)$

Because $N_{1}=N_{2}=2$ in Dataset 3 , the threshold $\theta$ and the weight $\boldsymbol{w}=\left(w_{1}, w_{2}\right)^{T}$ given by a PILD with $d_{p} \in\left\{N / N_{1}\right.$, $\left.-N / N_{2}\right\}$ or $d_{p} \in\{1,-1\}$ all are $\left(\theta, w_{1}, w_{2}\right)^{T}=(11 / 3,-4 / 3,-2 / 3)^{T}$, and the decision line equation by a PILD is $\pi_{l}: f\left(x_{1}, x_{2}\right)=\frac{11}{3}-\frac{4}{3} x_{1}-\frac{2}{3} x_{2}=11-4 x_{1}-2 x_{2}=0$. However, an FLD will be no longer in force for Dataset 3 because the scatter matrix $\boldsymbol{S}_{W}$ is singular.

The computational results of PILDs and FLDs for the three synthetic datasets are listed into Table $I$. We have found out the following facts from the table: $(A)$, In the case that the desired outputs are the fixed values $d_{p} \in\left\{N / N_{1}\right.$, $\left.-N / N_{2}\right\}$, a PILD is basically equal to an FLD with $\theta_{1}$, but there exist exceptions, e.g., $\alpha \neq 0$ in Example $1 ; \alpha \neq 1 / 2$ in Example 2, and in Example 3, a PILD is able to work normally, but an FLD not. $(B)$, If the desired outputs are the conventional values $d_{p} \in\{1,-1\}$, the normalized weight vectors given by PILDs and FLDs are the same, but the thresholds obtained by them two are different. Even more, the active regions of $\alpha$ determined by PILDs are wider than the ones by FLDs, which means that PILDs may be more robust than FLDs. In other words, the $(m+1)$-by- $(m+1)$ matrix $\tilde{\boldsymbol{X}}^{T} \tilde{\boldsymbol{X}}$ may not be so easily singular as the $m$-by- $m \boldsymbol{S}_{W} .(C)$ The class signs must be taken into consideration for calculating $\boldsymbol{S}_{W}$, but not for $\tilde{\boldsymbol{X}}^{T} \tilde{\boldsymbol{X}}$. We can still find such reverse cases that $\tilde{\boldsymbol{X}}^{T} \tilde{\boldsymbol{X}}$ are singular but $\boldsymbol{S}_{W}$ not, for

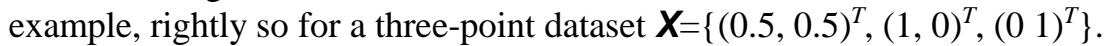

The conditions that a PILD and an FLD are actually equivalent are: (a) the desired outputs are the fixed values $d_{p} \in\left\{N / N_{1},-N / N_{2}\right\}$ for a PILD and the threshold is the totally projected mean $\theta_{1}=-\mu_{w}$ for an FLD; and (b) both the square matrix $\tilde{\boldsymbol{X}}^{T} \tilde{\boldsymbol{X}}$ in a PILD and the within-scatter matrix $\boldsymbol{S}_{W}$ in a FLD are nonsingular. In a word, we cannot conclude from the three examples above that a PILD is completely equivalent to an FLD with $\theta_{1}$ even if the desired outputs are assigned the specified values $\left\{N / N_{1},-N / N_{2}\right\}$.

\subsection{Relation to Fisher Linear Discriminant}

The subsection will give the detailed proof of relationship between PILDs and FLDs. We lay the special stress on comparing the explicit formulas of $\theta$ and $\boldsymbol{w}$ given by them two, and discuss the following two cases: (A) fixedly desire outputs $d_{p} \in\left\{N / N_{1},-\mathrm{N} / N_{2}\right\}, d_{p} \in\{\varphi,-\varphi\}$ including $d_{p} \in\{1,-1\}$, and $(B)$ changeably desired outputs $\boldsymbol{d}=\left(d_{1}, \ldots, d_{N}\right)^{T}$, subject to $d_{p} \neq d_{q}$ if $p \neq q$.

\subsubsection{Fixedly Desired Outputs}

\section{A. In Inverse Proportion to the Number of Within-Class Samples}

Without loss of generality, let the desired outputs be $d_{p}=N / N_{1}$ for $\boldsymbol{x}_{p} \in \omega_{1}$ and $d_{p}=-N / N_{2}$ for $\boldsymbol{x}_{p} \in \omega_{2}$. In the ideal case of $J(\theta, \boldsymbol{w})=\|\theta \mathbf{1}+\boldsymbol{X} \boldsymbol{w}-\boldsymbol{d}\|^{2}=0$, the explicit formulas of the $\theta$ and $\boldsymbol{w}$ given by a PILD are (Duda, Hart, \& Stork, 2000)

$$
\begin{aligned}
& \theta=\frac{1}{N}\left(\sum_{\boldsymbol{x}_{p} \in \omega_{1}} \frac{N}{N_{1}}-\sum_{\boldsymbol{x}_{p} \in \omega_{2}} \frac{N}{N_{2}}\right)-\frac{1}{N} \boldsymbol{w}^{T}\left(N_{1} \boldsymbol{\mu}_{1}+N_{2} \boldsymbol{\mu}_{2}\right)=-\frac{1}{N} \boldsymbol{w}^{T}\left(N_{1} \boldsymbol{\mu}_{1}+N_{2} \boldsymbol{\mu}_{2}\right)=-\mu_{\boldsymbol{w}}=\theta_{1} \\
& \boldsymbol{w}=\left(N-\frac{N_{1} N_{2}}{N}\left(\boldsymbol{\mu}_{1}-\boldsymbol{\mu}_{2}\right)^{T} \boldsymbol{w}\right) \boldsymbol{S}_{W}^{-1}\left(\boldsymbol{\mu}_{1}-\boldsymbol{\mu}_{2}\right)=\alpha \boldsymbol{S}_{W}^{-1}\left(\boldsymbol{\mu}_{1}-\boldsymbol{\mu}_{2}\right) \propto \boldsymbol{S}_{W}^{-1}\left(\boldsymbol{\mu}_{1}-\boldsymbol{\mu}_{2}\right)
\end{aligned}
$$

Duda, Hart, \& Stork (2000) had proved that the normalized weight $\boldsymbol{w}$ by a PILD with the desired outputs $d_{p} \in\left\{N / N_{1},-N / N_{2}\right\}$ is equivalent to the one by an FLD with the TPM threshold $\theta_{1}$. The two weight vectors are formally marked as $\boldsymbol{w}_{P I L D}=\alpha N \boldsymbol{S}_{W}^{-1}\left(\boldsymbol{\mu}_{1}-\boldsymbol{\mu}_{2}\right)$ and $\boldsymbol{w}_{F L D}=\boldsymbol{S}_{W}^{-1}\left(\boldsymbol{\mu}_{1}-\boldsymbol{\mu}_{2}\right)$. Obviously, the two normalized vectors are the same, 
i.e., $\boldsymbol{W}_{P I L D}=\boldsymbol{W}_{F L D}$. Note that the allocated values $d_{p} \in\left\{N / N_{1},-N / N_{2}\right\}$ are only a special case to meet Eq. (8).

Three examples in Subsection 3.1 have shown that even if in the case of $d_{p} \in\left\{N / N_{1},-N / N_{2}\right\}$, a PILD is not completely equivalent to an FLD with $\theta_{1}$. Or in more accurate words, they two are partly equivalent. Therefore, the biggest significance of proof of their relationship in the textbook by Duda, Hart, \& Stork (2000) seems to more establish the connection between them two than to demonstrate the equivalency, namely in name but not in reality.

\section{B. Equal to Some Artificial Constants}

Let $d_{p}=\varphi$ for $\boldsymbol{x}_{p} \in \omega_{1}$ and $d_{p}=-\varphi$ for $\boldsymbol{x}_{p} \in \omega_{2}$, in the ideal cases of $J(\theta, \boldsymbol{w})=\|\theta \mathbf{\theta}+\boldsymbol{X} \boldsymbol{w}-\boldsymbol{d}\|^{2}=0$, the explicit formulas of the $\theta$ and $\boldsymbol{w}$ given by a PILD are (Duda, Hart, $\&$ Stork, 2000)

$$
\begin{aligned}
& \theta=\frac{1}{N}\left(\sum_{\boldsymbol{x}_{p} \in \omega_{1}} \varphi-\sum_{\boldsymbol{x}_{p} \in \omega_{2}} \varphi\right)-\frac{1}{N} \boldsymbol{w}^{T}\left(N_{1} \boldsymbol{\mu}_{1}+N_{2} \boldsymbol{\mu}_{2}\right)=\frac{N_{1}-N_{2}}{N} \varphi-\frac{1}{N} \boldsymbol{w}^{T}\left(N_{1} \boldsymbol{\mu}_{1}+N_{2} \boldsymbol{\mu}_{2}\right)=\theta_{3.0} \\
& \boldsymbol{w}=\frac{N_{1} N_{2}}{N}\left(2 \varphi-\left(\boldsymbol{\mu}_{1}-\boldsymbol{\mu}_{2}\right)^{T} \boldsymbol{w}\right) \boldsymbol{S}_{W}^{-1}\left(\boldsymbol{\mu}_{1}-\boldsymbol{\mu}_{2}\right) \propto \boldsymbol{S}_{W}^{-1}\left(\boldsymbol{\mu}_{1}-\boldsymbol{\mu}_{2}\right)
\end{aligned}
$$

Especially, if $d_{p} \in\{1,-1\}$, which is the most common coding mode (Koford \& Groner, 1966; Elozondo, 2006; Escalera et al., 2008), Eqs. (10) and (11) become (Duda, Hart, \& Stork, 2000)

$$
\begin{gathered}
\theta=\frac{N_{1}-N_{2}}{N}-\frac{1}{N} \boldsymbol{w}^{T}\left(N_{1} \boldsymbol{\mu}_{1}+N_{2} \boldsymbol{\mu}_{2}\right)=\frac{N_{1}-N_{2}}{N}+\theta_{1}=\theta_{3} \\
\boldsymbol{w}=\frac{N_{1} N_{2}}{N}\left(2-\left(\boldsymbol{\mu}_{1}-\boldsymbol{\mu}_{2}\right)^{T} \boldsymbol{w}\right) \boldsymbol{S}_{W}^{-1}\left(\boldsymbol{\mu}_{1}-\boldsymbol{\mu}_{2}\right) \propto \boldsymbol{S}_{W}^{-1}\left(\boldsymbol{\mu}_{1}-\boldsymbol{\mu}_{2}\right)
\end{gathered}
$$

Here, a useful and basic threshold $\theta_{3}$ in Eq. (12) comes into being, which is yet size-related and almost as simple as $\theta_{1}$ in computation, called the $\mathrm{MS}^{2} \mathrm{E}$ threshold hereafter. Especially, if $N_{1}=N_{2}$, then $\theta_{3}=\theta_{2}=\theta_{1}$.

We assert that under the circumstances of the fixedly desired outputs $d_{p} \in\{\varphi,-\varphi\}$ including $d_{p} \in\{1,-1\}$, the normalized weight vector $\boldsymbol{w}$ given by a PILD is equivalent to the one by an FLD, or formally marked as $\boldsymbol{w}_{P I L D}=\boldsymbol{W}_{F L D}$, but the thresholds not, i.e., $\theta_{3} \neq \theta_{1}$ as well as $\theta_{3.0} \neq \theta_{1}$. Therefore, a PILD with $d_{p} \in\{\varphi,-\varphi\}$ is partly equivalent to an FLD with $\theta_{1}$ only.

The TPM thresholds $\theta_{1}$ are merely an approximation of the $\mathrm{MS}^{2} \mathrm{E}$ thresholds $\theta_{3}$ in the general cases of $N_{1} \neq N_{2}$. Because there exists the magnitude difference of $\left(N_{1}-N_{2}\right) / N$ between $\theta_{3}$ and $\theta_{1}$, the often-used thresholds $\theta_{1}$ perform poor in OCAs for the size-imbalanced datasets. If $d_{p} \in\{\varphi,-\varphi\}$ including $d_{p} \in\{1,-1\}$, the larger the difference between $N_{1}$ and $N_{2}$, the larger the difference between OERs of PILDs and FLDs with $\theta_{1}$ will result to.

\subsubsection{Changeably Desired Outputs}

Let the desired outputs be the actually algebraic Euclidean distances $d_{p}=\theta+\boldsymbol{w}^{T} \boldsymbol{x}_{p}(p=1, \ldots, N)$, and $d_{p} \neq d_{q}$ if $p \neq q$ in general. The explicit formulas of $\theta$ and $\boldsymbol{w}$ given by a PILD are

$$
\begin{aligned}
& \theta=\frac{1}{N} \sum_{p=1}^{N} d_{p}-\frac{1}{N} \boldsymbol{w}^{T}\left(N_{1} \boldsymbol{\mu}_{1}+N_{2} \boldsymbol{\mu}_{2}\right)=\frac{1}{N} \sum_{p=1}^{N} d_{p}+\theta_{1}=\theta_{4} \\
& \boldsymbol{w}=\boldsymbol{S}_{W}^{-1}\left(\sum_{p=1}^{N} d_{p} \boldsymbol{x}_{p}-\frac{1}{N}\left(\sum_{p=1}^{N} d_{p}\right)\left(N_{1} \boldsymbol{\mu}_{1}+N_{2} \boldsymbol{\mu}_{2}\right)-\frac{N_{1} N_{2}}{N}\left(\boldsymbol{\mu}_{1}-\boldsymbol{\mu}_{2}\right)\left(\boldsymbol{\mu}_{1}-\boldsymbol{\mu}_{2}\right)^{T} \boldsymbol{w}\right)
\end{aligned}
$$

The threshold $\theta_{4}$ is new, called the generally $\mathrm{MS}^{2} \mathrm{E}$ threshold. Let us make a comparison between Eqs (14) and (10). Obviously, the TPM threshold $\theta_{1}$ is only a special case of the generally MS2E threshold $\theta_{4}$ when $\sum_{p=1}^{N} d_{p}=0$. In other words, the TPM threshold is only a truncation of the generally MS2E threshold and thus behaves poor in OCAs (Gao, Ding, \& Zhu, 2014). Furthermore, Eq. (15) cannot be generally changed into the form of $\boldsymbol{w}=\alpha N \boldsymbol{S}_{W}^{-1}\left(\boldsymbol{\mu}_{1}-\boldsymbol{\mu}_{2}\right)$. In a word, when the desired outputs are changeable, not only the two thresholds, $\theta_{4}$ and $\theta_{1}$, but 
also the two normalized weights, $\boldsymbol{w}_{P I L D}$ and $\boldsymbol{w}_{F L D}$, have nothing in common, formally marked as $\left(\theta_{4}, \boldsymbol{w}\right)_{P I L D} \neq\left(\theta_{1}, \boldsymbol{w}\right)_{F L D}$. It must be stressed that the final threshold $\theta_{4}$ can only be calculated by using $\left(\theta_{4}, \boldsymbol{w}\right)^{T}=\left(\tilde{\boldsymbol{X}}^{T} \tilde{\boldsymbol{X}}\right)^{-1} \tilde{\boldsymbol{X}}^{T} \boldsymbol{d}$ in Eq. (2), but not directly by the explicit Eq. (14), because it is difficult to pre-designate the rational output vector $\boldsymbol{d}$. In addition, $\theta_{4}$ is not equal to $\theta_{3}$ unless all the training samples in two classes are just located on two parallel hyperplanes.

Equations (14) and (15) can be regarded as the generally fundamental explicit formulas of PILDs. Depending upon Eq. (14), we can set up the following relation

If $\sum_{p=1}^{N} d_{p}=0, \quad$ then $\theta_{4}=-\frac{1}{N} \boldsymbol{w}^{T}\left(N_{1} \boldsymbol{\mu}_{1}+N_{2} \boldsymbol{\mu}_{2}\right)=\theta_{1}$

The necessary prerequisite of $\theta_{4}=\theta_{1}$ is the zero sum of desired outputs. Here, two arbitrarily desired outputs need not be equal even if in the same class. If $d_{p} \in\left\{N / N_{1},-N / N_{2}\right\}$ or if $d_{p} \in\{\varphi,-\varphi\}$ or if $d_{p} \in\{1,-1\}$ in the cases of $N_{1}=N_{2}$, the relation $\sum_{p=1}^{N} d_{p}=0$ naturally meets, and thus Eq. (16) holds water.

We can get a precondition: if the sum of the first two terms in the right side of Eq. (15) is in the direction $\boldsymbol{\mu}_{1}-\boldsymbol{\mu}_{2}$, then the weight vector $\boldsymbol{w}$ will be in the direction $\boldsymbol{S}_{W}^{-1}\left(\boldsymbol{\mu}_{1}-\boldsymbol{\mu}_{2}\right)$, i.e.,

$$
\begin{aligned}
& \text { If } \sum_{p=1}^{N} d_{p} \boldsymbol{x}_{p}-\frac{1}{N}\left(\sum_{p=1}^{N} d_{p}\right)\left(N_{1} \boldsymbol{\mu}_{1}+N_{2} \boldsymbol{\mu}_{2}\right)=\beta\left(\boldsymbol{\mu}_{1}-\boldsymbol{\mu}_{2}\right) \\
& \text { then } \boldsymbol{w}=\boldsymbol{S}_{W}^{-1}\left(\sum_{p=1}^{N} d_{p} \boldsymbol{x}_{p}-\frac{1}{N}\left(\sum_{p=1}^{N} d_{p}\right)\left(N_{1} \boldsymbol{\mu}_{1}+N_{2} \boldsymbol{\mu}_{2}\right)-\frac{N_{1} N_{2}}{N}\left(\boldsymbol{\mu}_{1}-\boldsymbol{\mu}_{2}\right)\left(\boldsymbol{\mu}_{1}-\boldsymbol{\mu}_{2}\right)^{T} \boldsymbol{w}\right)=\alpha \boldsymbol{S}_{W}^{-1}\left(\boldsymbol{\mu}_{1}-\boldsymbol{\mu}_{2}\right)
\end{aligned}
$$

In particular, if $d_{p} \in\left\{N / N_{1},-N / N_{2}\right\}, d_{p} \in\{\varphi,-\varphi\}$ or $d_{p} \in\{1,-1\}$, Eq. (15) degenerates to be Eqs. (9), (11) and (13), in order.

The demonstrations above tell us that the processing Rayleigh quotient function $J(\boldsymbol{w})=\frac{\boldsymbol{w} \boldsymbol{S}_{B} \boldsymbol{w}}{\boldsymbol{w} \boldsymbol{S}_{W} \boldsymbol{w}}$ in an FLD is in essence another expression of the processing $\mathrm{MS}^{2} \mathrm{E}$ criterion function $J(\theta, \boldsymbol{w})=\left\|\tilde{\boldsymbol{X}}(\theta, \boldsymbol{w})^{T}-\boldsymbol{d}\right\|^{2}$ in a PILD. Both PILDs and FLDs aim at looking for optimal projected directions as well as maximizing class margins. Therefore, we ought not to feel the mystique of FLDs from now on.

We should understand the following facts: In order to analytically calculate the $\theta$ and $\boldsymbol{w}$ by using a PILD, we have to allocate in advance each class with the fixedly desired outputs $d_{p} \in\left\{\varphi_{1},-\varphi_{2}\right\}$ for all $p$. However, once the $\theta$ and $\boldsymbol{W}$ are obtained accordingly, the real outputs $y_{p}=\theta+\boldsymbol{w}^{T} \boldsymbol{x}_{p}$ are not usually equal to the desired $d_{p}$, or in more accurate words, the presuppositions $d_{p}=\theta+\boldsymbol{w}^{T} \boldsymbol{x}_{p}$ do not certainly hold water. Therefore, the original intention to minimize the sum of squared errors lies in seeking the statistical optimization for all the training samples.

\section{Threshold Development and Optimization}

The demonstration in Section 3 tells us that the threshold $\theta$ and the weight vector $\boldsymbol{w}$ in a PILD may be respectively treated, i.e., they may be written into two explicit formulas in the corresponding forms in an FLD. We had pointed out (Gao, Ding, \& Zhu, 2014) that "The change of thresholds actually signifies the parallel movement of separating hyperplanes $\pi$, which finally influences the classification accuracies of FLDs." In the same way, the performance of a PILD can be also optimized by selecting an appropriate threshold.

Let the OCA be $\rho$, then the OER is $1-\rho$. To a certain extent, to minimize the processing sum-of-squared-error function $J(\theta, \boldsymbol{w})$ is equivalent to maximize the value $\rho$. The thresholds $\theta_{3}$ and $\theta_{4}$ are deduced by minimizing $\boldsymbol{J}(\theta$, $\boldsymbol{w})$ accordingly.

Without loss of generality, $\omega_{1}$ is called the minority class and $\omega_{2}$ the majority class in the binary-class problem $\left\{\omega_{1}, \omega_{2}\right\}$; the numbers of samples in the two classes are $N_{1}$ and $N_{2}$, and $N_{2}>N_{1}$. The imbalanced ratio $I R=N_{2} / N_{1}$ is often used to measure the imbalanced degree of sizes. In the previous work (Gao, Ding, \& Zhu, 2014), we had found that when the sizes are seriously unequal, say $N_{2}>>N_{1}$, the TPM thresholds $\theta_{1}$ are quite disadvantageous to the majority 
classes $\omega_{2}$ if purely based on the OCAs, and thus proposed a corrected formula of Eq. (4), that is

$$
\theta_{5}=-\boldsymbol{w}^{T}\left(\frac{N_{2} \boldsymbol{\mu}_{1}+N_{1} \boldsymbol{\mu}_{2}}{N}\right)=-\frac{N_{2} \mu_{w}^{(1)}+N_{1} \mu_{w}^{(2)}}{N_{1}+N_{2}}
$$

where $\mu_{w}^{(1)}$ and $\mu_{w}^{(2)}$ are two projected means in the direction $\boldsymbol{w}$.

Equation (18) is just in the opposite side of Eq. (4). The larger the value $I R=N_{k} / N_{j}$, the more the threshold $\theta_{5}$ will be close to the negative minority-class mean $-\mu_{\boldsymbol{w}}^{(1)}$, and thus the decision hyperplane $\pi$ : $\theta_{5}+\boldsymbol{w}^{T} \boldsymbol{X}=0$ will deviate to the minority side $\omega_{j}$. Therefore, a PILD or FLD classifier with $\theta_{5}$ will outperform the one with $\theta_{1}$ in the aspect of OCAs.

Size imbalance is merely the outward appearance of sample distribution, and region imbalance is the essence. In other words, it is the distributed regions that have an essential influence on the performance of classifiers. The sizebased values IRs used for expressing the imbalanced degrees of many existing datasets (Alcala-Fdez et al., 2011) are merely for the sake of simplicity, and none of the thresholds from $\theta_{1}$ to $\theta_{5}$ takes the region imbalances into account.

In order to measure the imbalanced degree of distributed regions, the projected standard deviations $\sigma_{\boldsymbol{w}}^{(1)}$ and $\sigma_{w}^{(2)}$ first get into our sight line. Imitating the production of $\theta_{1}$ in Eq. (4), we have:

$$
\theta_{6}=-\frac{\sigma_{w}^{(1)} \mu_{w}^{(1)}+\sigma_{w}^{(2)} \mu_{w}^{(2)}}{\sigma_{w}^{(1)}+\sigma_{w}^{(2)}}
$$

Obviously, the threshold $\theta_{6}$ is more advantageous to the class with the narrower distributed region than to the side with the wider region as the $\theta_{1}$ does.

Besides the projected standard deviations, we can still employ the totally unbiased absolute deviations $\delta_{w}^{(1)}$ and $\delta_{\boldsymbol{w}}^{(2)}$, the averagely absolute deviations $\bar{\delta}_{\boldsymbol{w}}^{(1)}$ and $\bar{\delta}_{\boldsymbol{w}}^{(2)}$, the locally interval unbiased absolute deviations $\delta_{\boldsymbol{w}_{-} \text {in }}^{(1)}$ and $\delta_{\mathbf{w}_{-} \text {in }}^{(2)}$, and the locally interval average absolute deviations $\bar{\delta}_{\mathbf{w}_{-} i n}^{(1)}$ and $\bar{\delta}_{\boldsymbol{w}_{-} i n}^{(2)}$. The latter two pairs are determined by the samples within the projected interval $\left.\mid \mu_{w}^{(1)}, \mu_{w}^{(2)}\right\rfloor$. Please see our previous work (Gao, Ding, \& Zhu, 2014) for the detailed computation.

Similar to the form of $\theta_{5}$ in Eq. (18), we directly employ the 5 following empirical thresholds in our work (Gao, Ding, \& Zhu, 2014) so as to have a great choice, summarized in Table II. The reason to use the thresholds $\theta_{7}$ and $\theta_{11}$ is that they are more advantageous to the sides with the wider distributed regions than to the ones with the narrower. Note that the opposites of from $\theta_{7}$ to $\theta_{11}$ can be also developed, omitted here because they are disadvantageous to the improvement of OCAs.

The three thresholds, $\theta_{3}, \theta_{4}$ and $\theta_{6}$, are newly developed. The 11 thresholds above have their adaptive ranges. In the final analysis, the threshold with the highest OCA is singled out from among the 11 ones as the optimal. When two or more thresholds have equal OCAs, they should be given a preferential sequence. We empirically rank the 11 thresholds in the order $\theta_{8}, \theta_{9}, \theta_{7}, \theta_{5}, \theta_{2}, \theta_{11}, \theta_{10}, \theta_{6}, \theta_{4}, \theta_{3}$ and $\theta_{1}$. The optimal threshold $\theta_{\text {opt }}$ is given by:

$$
\theta_{\text {opt }}=\theta_{r}, \quad \text { if } \rho\left(\theta_{r}\right)=\max _{q=1}^{11} \rho\left(\theta_{q}\right)
$$

where $\rho\left(\theta_{q}\right)$ is the OCA of a PILD with the $q^{\text {th }}$ threshold $\theta_{q}$ for the binary-class training problem $\left\{\omega_{1}, \omega_{2}\right\}$.

Table III exemplifies the computational results of sizes, region-related values and thresholds for a synthetic 1-dimensional binary-class dataset: $\boldsymbol{X}^{(1)}=\{-2,2,3\} ; \boldsymbol{X}^{(2)}=\{4,4.25,4.75,7\}$. Directly by Eq. (2), we have $\theta_{4}=0.81$ and $\boldsymbol{w}=-0.29$. In accordance with the others, they are normalized as $\theta_{4}=0.81 / 0.29=2.80$ and $\boldsymbol{w}=-1.0$. Ten among the first 11 thresholds can be directly calculated either by sizes or by deviations, but $\theta_{4}$ cannot alone. Apparently, if the decision formula is $\theta+\boldsymbol{w}^{T} \boldsymbol{x}=\theta-x=0$, the PILDs with $\theta_{2}, \theta_{4}, \theta_{5}$ and $\theta_{6}$ fail to solve the linearly separable problem.

The meaning of the work in the section is how to improve the overall accuracies of PILDs with the help of multiple thresholds. Just through the proof of relationship between a PILD and an FLD, the explicit threshold and weight formulae are written, and therefore the performance of a PILD can be optimized by choosing an appropriate threshold. It must be stressed that the above-mentioned thresholds are based on the targeting OCAs. Besides, the available targeting criterion functions include the AUCs (Huang \& Ling, 2005; Wang \& Yao, 2013; Hand \& Till, 2001; Bradley, 1997; Adams \& Hand, 1999), the geometric mean (G-mean) accuracies $\bar{\rho}_{g m}=\sqrt{\rho_{1} \rho_{2}}$ (Fernandez et al., 
2008; Barua et al., 2014; Castro \& Braga, 2013), and the simple mean (S-mean) accuracies $\bar{\rho}=\left(\rho_{1}+\rho_{2}\right) / 2$ (Galar et al., 2012; Cano, Zafra, \& Ventura, 2013; Galar et al., 2013). If the targeting criterions change, the optimal thresholds will change accordingly. Not only that, more thresholds, e.g., the opposites of from $\theta_{8}$ to $\theta_{11}$, may be developed and possibly display good performances.

\section{Iterative Learning and Sample Decomposition}

This section focuses on improving the OCAs of PILDs by using two following learning strategies: $(a)$ iteration with changeably desired outputs and $(b)$ within-class sample decomposition.

Without loss of generality, the discussions below are yet limited to the binary-class training dataset $\boldsymbol{X}^{(1,2)}=\left\{\boldsymbol{X}^{(1)}\right.$, $\left.\boldsymbol{X}^{(2)}\right\}$. Let the desired output be $d_{p}>0$ for a sample $\boldsymbol{X}_{p}$ belonging to the positive class $\omega_{1}$, and $d_{p}<0$ for $\boldsymbol{X}_{p}$ to the negative class $\omega_{2}$.

The value $y_{p}=f\left(\boldsymbol{x}_{p}\right)=\theta+\boldsymbol{w}^{T} \boldsymbol{x}_{p}$ actually expresses the algebraic Euclidean distance from the point $\boldsymbol{x}_{p}$ to the decision hyperplane $\pi_{l}: \theta+\boldsymbol{w}^{T} \boldsymbol{X}=0$. Equation (14) about $\theta_{4}$ enlightens us that an optimal threshold can be calculated directly by using the changeably desired outputs and two projected means. Starting from that, an iterative learning strategy of PILDs can be presented in order to further optimize both the thresholds $\theta$ and the weights $\boldsymbol{w}$.

\section{(A) Iterative Learning with Changeably Desired Outputs}

Our main idea is as follows: Once the initial optimal threshold $\theta_{o p t}(0)$ and the weight vector $\boldsymbol{w}(0)$ are obtained by a PILD at the epoch $\tau=0$, all the actually algebraic Euclidean distances $y_{p}(0)=\theta_{\text {opt }}(0)+\boldsymbol{w}(0)^{T} \boldsymbol{x}_{p}(p=1, \ldots, N)$ are calculated accordingly. Of course, the initial targeting OCA $\rho(0)$ can be determined consequently. At the epoch $\tau+1$, the desired outputs $d_{p}(\tau+1)$ can be replaced by the actual distances $y_{p}(\tau)=\theta_{o p t}(\tau)+\boldsymbol{W}(\tau)^{T} \boldsymbol{X}_{p}$ gotten at the preceding step $\tau$, i.e., $d_{p}(\tau+1) \leftarrow y_{p}(\tau)$. Consequently, the new thresholds $\theta_{q}(\tau+1)(q=1, \ldots, 11$,opt $)$ and weight $\boldsymbol{w}(\tau+1)$ are brought forth. In that approach, the iterative learning strategy of PILD is realized. The resulting $\theta_{q}(\tau+1)$ and $\boldsymbol{w}(\tau+1)$ do not correspond to any one given by an FLD.

In order to execute the iterative learning procedure, a key problem to be solved is how to re-allocate the desired outputs of mislabeled samples at the $\tau+1^{\text {th }}$ epoch. A good action is to make the desired outputs of the misclassified positive/negative-class samples mandatorily equal to half of the smallest/largest algebraic distances of the correct positive/negative-class samples, to the current separating hyperplane $\pi: \theta_{o p t}(\tau)+\boldsymbol{w}^{T}(\tau) \boldsymbol{X}=0$. Therefore, we need determine in advance the smallest algebraic distance $d_{\min }^{(1)}(\tau)>0$ for the positive class $\omega_{1}$ and the largest $d_{\max }^{(2)}(\tau)<0$ for the negative class $\omega_{2}$. The pair $\left\{d_{\min }^{(1)}(\tau), d_{\max }^{(2)}(\tau)\right\}$ is calculated below:

$$
\left\{\begin{array}{l}
d_{\min }^{(1)}(\tau)=\min _{p=1}^{N_{1}}\left(y_{p}(\tau)\right), \quad \text { if } \quad \boldsymbol{x}_{p} \in \omega_{1} \text { and } y_{p}(\tau)=\theta_{\text {opt }}(\tau)+\boldsymbol{w}^{T}(\tau) \boldsymbol{x}_{p}>0 \\
d_{\max }^{(2)}(\tau)=\max _{p=N_{1}+1}^{N_{1}+N_{2}}\left(y_{p}(\tau)\right), \quad \text { if } \quad \boldsymbol{x}_{p} \in \omega_{2} \text { and } y_{p}(\tau)=\theta_{\text {opt }}(\tau)+\boldsymbol{w}^{T}(\tau) \boldsymbol{x}_{p}<0
\end{array}\right.
$$

Figure 1 illustrates such a case for re-allocating the desired output of a mislabeled negative-class sample $\boldsymbol{x}_{p}$. At the epoch $\tau$, the sample $\boldsymbol{x}_{p}$ was assigned a negatively desired output $d_{p}(\tau)<0$, but has a positively real algebraic output $y_{p}(\tau)(>0)$. At the epoch $\tau+1$, the $\boldsymbol{x}_{p}$ is re-allocated a negatively desired output $d_{p}(\tau+1)=d_{\max }^{(2)}(\tau) / 2 \quad(<0)$, and thus is re-identified.

The desired output $d_{p}(\tau+1)$ for the training sample $\boldsymbol{x}_{p}$ is re-allocated as follows:

$\left\{\begin{array}{lll}d_{p}^{(1)}(\tau+1)=d_{\min }^{(1)}(\tau) / 2 \text { if } y_{p}(\tau)<0 ; & \text { otherwise } d_{p}^{(1)}(\tau+1)=y_{p}(\tau), \text { for } \boldsymbol{x}_{p} \in \omega_{1} . \\ d_{p}^{(2)}(\tau+1)=d_{\max }^{(2)}(\tau) / 2 \text { if } y_{p}(\tau)>0 ; & \text { otherwise } d_{p}^{(2)}(\tau+1)=y_{p}(\tau), \text { for } \boldsymbol{x}_{p} \in \omega_{2} .\end{array}\right.$

In the way, all the desired outputs of training samples are updated before executing the $\tau+1^{\text {th }}$ epoch learning, i.e., $\boldsymbol{d}(\tau+1)=\left(d_{1}^{(1)}(\tau+1), \cdots, d_{N_{1}}^{(1)}(\tau+1), d_{N_{1}+1}^{(2)}(\tau+1), \cdots, d_{N_{1}+N_{2}}^{(2)}(\tau+1)\right)^{T}$.

To summarize, the basic iterative formula is 


$$
\left(\theta_{4}(\tau+1), \boldsymbol{w}(\tau+1)\right)^{T}=\left(\left(\tilde{\boldsymbol{X}}^{(1,2)}(\tau+1)\right)^{T}\left(\tilde{\boldsymbol{X}}^{(1,2)}(\tau+1)\right)\right)^{-1}\left(\tilde{\boldsymbol{X}}^{(1,2)}(\tau+1)\right)^{T} \boldsymbol{d}(\tau+1)
$$

Equation (23) embodies not only the update of desired outputs but also the decomposition of samples. This type of iterative learning is in essence different from the conventional iterative format $\boldsymbol{w}(\tau+1)=\boldsymbol{W}(\tau)+\eta \nabla \boldsymbol{W}(\tau)$ in the aspect that the step-size factor is not required during learning.

As soon as the threshold $\theta_{4}(\tau+1)$ and the weight $\boldsymbol{w}(\tau+1)$ are calculated, the other 11 thresholds are obtained accordingly. Whether the $\theta_{q}(\tau+1)$ and the $\boldsymbol{W}(\tau+1)$ are updated depends upon the up-and-down situation of OCAs for the original training dataset $\boldsymbol{X}^{(1,2)}(0)$. If the PILD has a higher OCA at the epoch $\tau+1$ than that at the epoch $\tau$, say $\rho(\tau+1)>\rho(\tau)$, the iterative result is accepted and thus the previous pair $\left\{\theta_{o p t}(\tau), \boldsymbol{w}(\tau)\right\}$ is updated by the current pair $\left\{\theta_{o p t}(\tau+1), \boldsymbol{W}(\tau+1)\right\}$; otherwise rejected. Under the restriction of improvement of OCAs, the iterative epochs will be limited, and the divergent risk can be avoided. In other words, if the current OCA $\rho(\tau+1)$ is worse than the previous $\rho(\tau)$, the present pair $\left\{\theta_{o p t}(\tau+1), \boldsymbol{W}(\tau+1)\right\}$ is abandoned. In particular, if the current OCA is $\rho(\tau)=100 \%$, the iterative process ends.

According to the conditional risk minimization rule, the iterative learning process may be terminated when the OCA for the current training dataset comes down. In practice, we empirically allow a little decrease of OCAs. Our practice is to temporarily record every 5 successive pairs of thresholds and weights from $\left\{\theta_{o p t}(\tau), \boldsymbol{W}(\tau)\right\}$ until $\left\{\theta_{o p t}(\tau-4)\right.$, $\boldsymbol{w}(\tau-4)\}$ and try to choose $\left\{\theta_{\text {opt }}(\tau-4), \boldsymbol{w}(\tau-4)\right\}$ as the resulting. If the 5 succeeding pairs of $\left\{\theta_{o p t}\right.$, $\left.\boldsymbol{w}\right\}$ keep the OCAs going down, i.e., $\rho(\tau)<\rho(\tau-1)<\ldots<\rho(\tau-4)$, the iterative process terminates; otherwise continues. As a result, the optimal pair $\left\{\theta_{\text {opt }}(\tau-4), \boldsymbol{W}(\tau-4)\right\}$ as well as the other thresholds are saved as the final.

\section{(B) Within-class Sample Decomposition}

Next we carry out the decomposition of the training subset $\boldsymbol{X}^{(1,2)}$. A basic fact is that the relation $\mu_{w}^{(1)}(\tau)<\theta_{q}(\tau)<\mu_{w}^{(2)}(\tau)$ always meets. In order words, the samples outside the closed interval $\left\lfloor\mu_{w}^{(1)}(\tau), \mu_{w}^{(2)}(\tau)\right\rfloor$, called the external samples, will not have influence on the OCA anymore, regardless of whether they are identified. Therefore, the external samples don't have to attend in the follow-up learning. It is considered that the lower-limit ratio of size to dimensionality in the subclass $\boldsymbol{X}^{(j)}(j=1,2)$ should be over $N_{j}(\tau) / m>4$ for the trustworthy design of linear classifiers based on the conditional risk minimization (Fukunaga \& Hayes, 1989). If the ratio $N_{j}(\tau) / m$ is lager enough, $\boldsymbol{X}^{(\mathrm{j})}$ can be decomposed further. Our action is that if $N_{j}(\tau) / m \geq 8$, the samples from $\boldsymbol{X}^{(j)}(\tau)$ in class $\omega_{j}$ within the interval $\left[\mu_{w}^{(1)}(\tau), \mu_{w}^{(2)}(\tau)\right]$ form a part of the new training subset $\boldsymbol{X}^{(1,2)}(\tau+1)$; otherwise gotten rid of. The thresholds $\theta_{q}(\tau+1)$ and the weight $\boldsymbol{w}(\tau+1)$ are recalculated by the reduced samples.

Once the current dataset $\boldsymbol{X}^{(1,2)}(\tau)$ is implemented the sample decomposition, the following action returns to the iterative learning with changeably desired outputs again. Because only about a half of the current training samples will be left at every decomposition turn, the number of times to do decomposition will be quite limited. Comparatively speaking, the final number of times of sample decomposition is far fewer than the number of iterative learning epochs with changeably desired outputs.

Sometimes the constant column 1 will lead to an unduly large or small element in the square matrix $\left(\tilde{\boldsymbol{X}}^{(1,2)}\right)^{T} \tilde{\boldsymbol{X}}^{(1,2)}$, which may thus become singular or ill-conditioned. In order to guard against the case, we can multiply the column $\mathbf{1}$ by a total size coefficient $\gamma$ equal to the total mean of $\boldsymbol{X}$, i.e., $\gamma=$ mean $($ mean $(\boldsymbol{X}))$ in Matlab language, to become $\tilde{\boldsymbol{X}}^{(1,2)}=\left(\gamma \mathbf{1}, \boldsymbol{X}^{(1,2)}\right)$. In particular, let $\gamma=0.1$ if a dataset is normalized or dimensionality-reduced by principal component analysis (PCA).

The iterative learning strategy of a PILD is quite different from the one of an FLD (Gao, Ding, \& Zhu, 2014) in the following aspects: $(A)$ The targeting evaluation criterion, i.e., the overall accuracy by means of the classified or misclassified judgment, is directly used to update the desired outputs of samples by Eqs. (21) and (22). (B) The processing evaluation criterion, i.e., the $\mathrm{MS}^{2} \mathrm{E}$ function, is employed to calculate the threshold $\theta_{4}$ and the weight vector $\boldsymbol{w}$ simultaneously in multiple iterative epochs by Eq. (23), regardless whether the dataset is decomposed or not. (C) The iteration strategy and the decomposition operation are simultaneously employed, and the iterative learning can be carried out independently.

\section{Experimental Results}




\subsection{Sonar Recognition}

The classical Sonar dataset has $N_{1}=49$ and $N_{2}=55$ training samples in two subclasses $\boldsymbol{X}^{(1)}$ and $\boldsymbol{X}^{(2)}$, respectively, in the 60-dimensional space (Asuncion \& Newman, 2010; Gormann, \& Sejnowski, 1988; Roy, Govil, \& Miranda, 1995). $I R=55 / 49=1.12$, which indicates that the dataset is almost balanced in size. The total size coefficient for the constant column 1 is $\gamma=0.2773$. Fig. 2 gives the changes of OERs, min and max algebraic Euclidean distances $\left\{d_{\min }^{(1)}(\tau), d_{\max }^{(2)}(\tau)\right\}$, optimal thresholds $\theta_{\text {opt }}$ by a PILD with the iterative learning strategy. The pairs $\left\{d_{\min }^{(1)}(\tau), d_{\max }^{(2)}(\tau)\right\}$ change quite rough at the initial stage and gentle soon afterwards, from the initial $\left\{d_{\min }^{(1)}(0)=0.0561\right.$, $\left.d_{\text {max }}^{(2)}(0)=-0.0082\right\}$ to the final $\left\{d_{\min }^{(1)}(15)=2.68 \times 10^{-4}, d_{\max }^{(2)}(15)=-1.04 \times 10^{-5}\right\}$. It is interesting to note the change of indices of optimal threshold $\theta_{\text {opt }} \leftarrow \theta_{q}$, undergoing $\theta_{5}, \theta_{8}, \theta_{2}, \theta_{8}, \theta_{9}, \theta_{10}, \theta_{9}, \theta_{7}, \theta_{8}$ and $\theta_{11}$ in order. The learning time increases from $0.062 \mathrm{sec}$ at $\tau=0$ epoch to $0.187 \mathrm{sec}$ with $\tau=15$ epochs by a Personal Computer (PC) with 3.2G CPU and 4.0G RAM (the same below). The resulting OCA is $100 \%$ for the training set, though the OCA for the test set still with 104 samples almost keeps unchanged, i.e., 1-25/104=75.96\%. The AUC value for the test set is $83.03 \%$.

An outstanding characteristic of the iterative PILD is that no prior step-size factor is needed. The PILD_ $\theta_{o p t}$ achieves the overall training accuracy of $100 \%$ only with $\tau=15$ epochs due to the iterative learning with changeably desired outputs, which outperforms all the existing classifiers to our knowledge (Shen \& Li, 2010; Toh, Tran, \& Srinivasan, 2004), including multi-layer perceptrons (Gormann, \& Sejnowski, 1988; Roy, Govil, \& Miranda, 1995) and SVMs (Suykens et al., 2002; Jayadeva, Khemchandani, \& Chandra, 2007; Kim \& Scott, 2010).

\subsection{Adult Dataset}

The binary-class Adult problem is from the census bureau database of USA (Asuncion \& Newman, 2010). In the 14-dimensional space, there are 30,162 training and 15,060 test samples. $I R=22654: 7508=3.02$ for the training set, which implies that the degree of size imbalance is not serious.

The Adult data is firstly normalized and then let $\gamma=0.1$. The iterative learning process undergoes 5 iteration stages and 4 decomposition operations, and ends after 60 steps with $196.02 \mathrm{sec}$. Figure 3 shows the changes of OERs, optimal threshold $\theta_{\text {opt }}$ 's indices, and min and max algebraic distances, $d_{\min }^{(1)}$ and $d_{\max }^{(2)}$, in the first two iteration stages with one decomposition operation. The first iteration stage undergoes 31 epochs (not including the initial) and the optimal result happens at the $27^{\text {th }}$ epoch, which is signed by "Opt 1 " in Fig.3, because the following 4 OERs always come up. After the first decomposition operation, the PILD_ $\theta_{\text {opt }}$ only goes through 11 epochs at the $2^{\text {nd }}$ iteration stage and has sought out the optimal result at the $7^{\text {th }}$ epoch, signed by "Opt $t_{2}$ ".

According to Fig. 3, the Minimum and maximum algebraic Euclidean distance pairs $\left\{d_{\min }^{(1)}(\tau), d_{\max }^{(2)}(\tau)\right\}$ change violently; however, the optimal threshold $\theta_{\text {opt }}$ exchanges only between $\theta_{4}$ and $\theta_{5}$. Table $I V$ gives the detailed learning results of the iterative PILDs with all 5 iteration stages. Going throughout 4 decomposition operations, the numbers of learning samples become fewer and fewer, the mean intervals $\left[\mu_{1}, \mu_{2}\right]$ narrower and narrower, and the algebraic Euclidean distances $\left(d_{\min }^{(1)}(\tau), d_{\max }^{(2)}(\tau)\right)$ smaller and smaller. In fact, the learning result of PILDs with 80 samples at the $5^{\text {th }}$ iteration stage is unbelievable because the augmented squared matrix $\tilde{\boldsymbol{X}}^{T} \tilde{\boldsymbol{X}}$ is highly singular.

Through the iterative learning with changeably desired outputs and sample decomposition, the OCAs are respectively increased from $71.56 \%$ and $82.18 \%$ to $83.53 \%$ for the training set, and from $71.09 \%$ and $81.84 \%$ to $83.32 \%$ for the test set, given by from the initial PILD_ $\theta_{1}$ and PILD_ $\theta_{o p t}$ to the final PILD_ $\theta_{\text {opt }}$ with $\tau=60$ epochs. The resulting AUC values are respectively $88.64 \%$ and $88.44 \%$ for the training and the test set.

The other OCAs for the test set are $79.08 \%$ by the nearest-neighbor $(1-N N)$ classifier, $71.09 \%$ by an FLD_ $\theta_{1}$, $78.48 \%$ by an FLD_ $\theta_{2}$, and $81.78 \%$ by a PILD_ $\theta_{4}$ without iteration. Therefore, the iterative PILD with optimal threshold is quite effective.

\subsection{More Benchmark Datasets}

The experiments below are about the benchmark datasets in the UCI Machine Learning Repository (Asuncion \& Newman, 2010), KEEL Imbalanced Repository (Alcala-Fdez et al., 2011), and a 2-dimensional (2D) vowel data (Roy, Govil, \& Miranda, 1995). If only a data file is available at the website, the dataset is split into 2:1 ratio of training to 
test size and specified to each class in the original order of samples by the nearest integer rule. In order to make the matrix $\tilde{\boldsymbol{X}}^{T} \tilde{\boldsymbol{X}}$ reliably invertible, the Image segment dataset is reduced from 18 to 7 dimensions by PCA. For the original Splice dataset, four nominal features $\{A, C, G, T\}$ are encoded as $\{(0,0),(0,1),(1,0),(1,1)\}$ in binary, which thus forms the Splice- $b$ dataset. Table $V$ gives the numerical characteristics of 24 benchmark datasets listed in the $I R$ order. In the viewpoint of sizes, the Image segment, Letter, Optdigit, Pendigit datasets are or approximately balanced, because their $I R$ s are quite close to 1 . The Shuttle dataset has the highest imbalanced ratio, reaching $I R_{\max }=5684.67$.

Table VI gives the experimental results of iterative PILDs with different thresholds for the 24 benchmark test datasets, and Appendix $A$ gives the same results for the 24 corresponding benchmark training datasets. According to the table, the PILDs_ $\theta_{\text {opt }}$ have the highest OCAs, and get an average increase of $1.09-20.26 \%$ for the test sets, compared with the other PILDs_ $\theta_{q}(q=1, \ldots, 11)$. The largest average differences of OCAs happen between PILDs_ $\theta_{\text {opt }}$ and PILDs_ $\theta_{1}$. Obviously, the PILDs_ $\theta_{\text {opt }}$ have observably improved the OCAs for the 2D vowel, German credit, Shuttle and Yeast datasets.

Let us have a special analysis on the binary-class Abalone 19 dataset (Alcala-Fdez et al., 2011). The value $I R=$ $2761 / 21=131.48$ signifies that the imbalanced degree of sizes is a bit serious. No iteration is done because the OERs always go up in the first 5 epochs. The OCAs of the PILD with $\theta_{o p t}\left(=\theta_{3}\right)$ are $99.25 \%$ for the training and $99.21 \%$ for the test set, respectively. However, all 21 training and 11 test samples from the minority class are wholly misclassified. Such results are not satisfactory. The AUC value cannot help us anything to threshold optimization. If we go to another extreme to let $\theta_{o p t}=\theta_{1}$, all the samples in the minority class are classified, but 1042 training and 511 test samples in the majority class are mislabeled, i.e., the OCAs of $62.54 \%$ for the training and $63.29 \%$ for the test set only. Such results with too many misrecognized majority-class samples are not satisfactory yet. Here, we would rather choose $\theta_{5}$ as the optimal $\theta_{\text {opt }}$ on an average of OCA, G-mean, S-mean and AUC. At the moment, the OCAs are $90.69 \%$ and $93.10 \%$ for the two sets, and 9 training and 6 test samples in the minority class, 250 training samples in the majority class, are mislabeled. The issue deals with how to develop appropriately targeting criterion functions. The future work will devote to it.

According to Table $V I$, the TPM thresholds $\theta_{1}=-\mu_{w}$ behave poor if only based on OCAs. A striking contrast happens between the region-based $\theta_{6}$ and the size-based $\theta_{1}$. The $\theta_{6}$ in Eq. (19) is obtained only by simply taking the place of the size $N_{1}$ and $N_{2}$ in Eq. (4) with the standard deviations $\sigma_{\boldsymbol{w}}^{(1)}$ and $\sigma_{\boldsymbol{w}}^{(2)}$. However, the PILDs_ $\theta_{6}$ with the average OCA of $77.98 \%$ far outperform the PILDs_ $\theta_{1}$ with only the average OCA of $65.64 \%$ for the test datasets, which indubitably demonstrates that distributed regions are far more important than sample sizes. Under many circumstances, the thresholds $\theta_{4}$ and even $\theta_{3}$ behave relatively well, which tells us that the terms $\frac{1}{N} \sum_{p=1}^{N} d_{p}$ and $\frac{N_{1}-N_{2}}{N}$ have an important influence on the performance of PILDs and ought not to be truncated casually. Drawing inspiration from the results above, Fisher linear classifiers thus have more thresholds for selection besides the commonly used $\theta_{1}$ and $\theta_{2}$.

Next, we take the nonparametric paired signed-rank Wilcoxon test (Sheskin, 2006) to evaluate the performance of PILDs. The statistically significant differences in the pairs $\left\{\right.$ PILDs_ $\theta_{q}$, PILDs_ $\left.\theta_{r}\right\}(q>r)$ are tested by judging whether the hypotheses $H_{0}$ : PILDs_ $\theta_{q}=$ PILDs_ $\theta_{r}$ are accepted. Let $R_{q r}^{+}$be the positive rank sum in which PILDs_ $\theta_{q}$ outperform PILDs_ $\theta_{r}$, and $R_{q r}^{-}$the one for the opposite. The paired sums $\left\{R_{q r}^{+}, R_{q r}^{-}\right\}$are listed in Table VII for the 24 benchmark test datasets, which is a left-upper triangular matrix. Appendix $B$ lists the paired sums for the 24 corresponding benchmark training datasets.

The max number of valid datasets is $n_{d s}=24$, but $n_{d s}<24$ for the equal OCA cases. At the confidence level $\xi=0.05$, the allowable min rank sums, i.e., the allowable tested statistic variables, are $T=81$ for $n_{d s}=24, T=73$ for $n_{d s}=23, T=65$ for $n_{d s}=22, T=58$ for $n_{d s}=21$, etc (Sheskin, 2006). Whether the PILDs_ $\theta_{q}$ have a statistical difference from the PILDs_ $\theta_{r}$ depends upon whether the minimum differences $R_{q r}^{d i f}=\min \left\{R_{q r}^{+},\left|R_{q r}^{-}\right|\right\}$are smaller than the allowable sums $T$.

According to Table VII, we find the following facts:

(A) The hypotheses $H_{0}$ : PILDs_ $\theta_{o p t}=$ PILDs $\_\theta_{r}(r=1, \ldots, 11)$ are rejected, because the max value $R_{o p t, r}^{\text {dif }}=37$ for the 
test sets are far smaller than those from $T=81$ to $T=58$. In other words, PILDs_ $\theta_{o p t}$ outperform PILDs_ $\theta_{r}$ $(r=1, \ldots, 11)$.

(B) The hypotheses $H_{0}$ : PILDs_ $\theta_{q}=$ PILDs_ $\theta_{1}(q=2, \ldots, 11)$ are rejected, because the max value $R_{q, 1}^{\text {dif }}=30$ for the test sets is far smaller than those from $T=81$ to $T=34$ for $n_{d s}=17$. As a matter of fact, PILDs_ $\theta_{q}(q=2, \ldots, 11)$ apparently outperform PILDs_ $\theta_{1}$. In particular, the fact that PILDs_ $\theta_{6}$ observably outperform PILDs_ $\theta_{1}$ tells us again that distributed regions are more important than sample sizes to improve the OCAs of PILDs and FLDs.

(C) The hypothesis $H_{0}$ : PILDs_ $\theta_{4}=$ PILDs_ $\theta_{3}$ is accepted, because the paired value $\left\{R_{4,3}^{+}, R_{4,3}^{-}\right\}=\{6,0\}$ for $n_{d s}=3$ test sets only accounts for a small part of the 24 datasets. The result shows that the term $\frac{1}{N} \sum_{p=1}^{N} d_{p}$ in the $\theta_{4}$ can be approximated by $\frac{N_{1}-N_{2}}{N}$ in the $\theta_{3}$. Here, an important threshold $\theta_{3}$ by Eq. (12) is found, which is quite useful both for PILDs and for FLDs. The threshold $\theta_{3}$ may be simply acquired by adding the size-related term $\frac{N_{1}-N_{2}}{N}$ to the $\theta_{1}$.

Now, we compare the learning and generalization performances of iterative PILDs and FILDs (Gao, Ding, \& Zhu, 2014), with the optimal thresholds, as shown in Table VIII. Note that the thresholds in them two are not wholly equal and corresponding. We have found several such facts from the table: (A) PILDs are not certainly equivalent to FLDs. Among the 24 training results, the iterative PILDs_ $\theta_{\text {opt }}$ outperform the iterative FLDs_ $\theta_{12}$ for 16 ; on the contrary, the latter outperform the former for 7. Averagely speaking, the former has a higher OCA than the latter. $(B)$ PILDs_ $\theta_{o p t}$ may do multiple learning epochs both by iteration and by decomposition operation, e.g., 60 epochs for the Adult dataset, but FLDs_ $\theta_{12}$ iterate no more than 3 epochs merely by decomposition operation. Therefore, the former takes a longer runtime than the latter does. $(C)$ The combination of PILDs_ $\theta_{o p t}$ and FLDs_ $\theta_{o p t}$ can attain some higher OCAs.

To our knowledge, the PILDs_ $\theta_{\text {opt }}$ have reached the highest OCAs until now for some datasets, such as the 2D vowel, German credit, Splice and Yeast, even though compared with the well-known superior classifiers, e.g., neural networks and SVMs, in the literature (Suykens et al., 2002; Shen \& Li, 2010; Asuncion \& Newman, 2010; Roy, Govil, \& Miranda, 1995; Kim \& Scott, 2010; Li, Tsang, \& Zhou,. 2013).

\section{Conclusions and discussions}

This paper addresses the optimal learning and generalization problems of pseudo-inverse linear discriminants based on the processing $\mathrm{MS}^{2} \mathrm{E}$ and the targeting OCA criterion functions. There is little theoretical and practicable significance to prove the equivalency between a PILD and an FLD by using the designated outputs $\left\{N / N_{1},-N / N_{2}\right\}$. Even if so, three synthetic examples have indicated that a PILD is not equivalent to an FLD. Consequently, the most often-used TPM thresholds perform poor in OCAs. If all training samples of each class are mandatorily allocated a fixedly desired output, a PILD and an FLD are partly equivalent; otherwise nothing in common. By means of the conventionally desired outputs $\{1,-1\}$, a simple practicable $\mathrm{MS}^{2} \mathrm{E}$ threshold can be developed, which is only related to sizes. By assigning the changeably desired output to each sample, a generally explicit threshold, i.e., $\theta_{4}$, and an explicit weight vector, are hence obtained. Many size- and region-related thresholds are thus developed, and the 
optimal thresholds are singled out from among them according to the OCAs. With the help of the processing $\mathrm{MS}^{2} \mathrm{E}$ criterions and the actually algebraic distances, an iterative learning strategy of PILD is proposed. Three outstanding strong points of the iterative PILDs are (i) limited epochs, (ii) no in need of learning rate and (iii) without risk of divergence. Enormously experimental results for many real-world datasets have verified that the iterative PILDs with the optimal thresholds have good learning and generalization performances, and even reach the best OCAs for some datasets among the existing linear and nonlinear classifiers.

Starting from our previous work (Gao, Ding, \& Zhu, 2014), we have had several such developments and contributions: ( $a$ ) Some new thresholds have been developed by comparing the similarities and differences of PILDs and FLDs; $(b)$ An FLD is unable to utilize the processing minimum sum-of-squared error and changeably desired outputs to optimize the weight and threshold, but a PILD is able to; (c) An FLD cannot use the targeting OCA criterion function to evaluate the learning performance itself, but a PILD can.

Both optimal thresholds and optimal weight vectors will change if the targeting criterion functions change. For instance, in the field of data mining, the misclassification costs may be much larger for the minority-class samples than those for the majority-class ones, and the other targeting criterion functions, such as S-means, G-means, AUCs, will probably display their strong points. The future work will devote to the aspects. 


\section{Acknowledgments}

This work is funded by the National Science Foundation of China (NSFC) under Grant Nos. 21176077, 61272198 and 60675027, the High-Tech Development Program of China (863) under Grant No. 2006AA10Z315, and the Open Funding Project of the State Key Laboratory of Bioreactor Engineering. 


\section{References}

Adams, N. M., \& Hand, D. J. (1999). Comparing classifiers when the misallocation costs are uncertain. Pattern Recognition. 32(7): 1139-1147.

Alcala-Fdez, J., Fernandez, A., Luengo, J., Derrac, J., Garcia, S., Sanchez, L., \& Herrera, F. (2011). KEEL data-mining software tool: Data Set Repository [Online], Available: http://www.keel.es/imbalanced.php.

Asuncion, A., \& Newman, D. J. (2010). UCI Machine Learning Repository [Online]. Available: http://www.ics.uci.edu/mlearn/ MLRepository.html.

Barua, S., Islam, M. M., Yao, X., \& Murase, K. (2014). MWMOTE-majority weighted minority oversampling technique for imbalanced data set learning. IEEE Transactions on Knowledge and Data Engineering. 26(2): 405-425.

Belhumeur, P. N., Hespanha, J. P., \& Kriegman, D. J. (2007). Eigenfaces vs. Fisherfaces: Recognition using class specific linear projection. IEEE Transactions on Pattern Analysis and Machine Intelligence. 19(7): 711-720.

Billings, S. A., \& Lee, K. L. (2002). Nonlinear Fisher discriminant analysis using a minimum squared error cost function and the orthogonal least squares algorithm. Neural Networks. 15(2): 263-270.

Bradley, A. E. (1997). The use of the area under the ROC curve in the evaluation of machine learning algorithms. Pattern Recognition. 30(7): 1145-1159.

Cano, A., Zafra, A., \& Ventura, S. (2013). Weighted data gravitation classification for standard and imbalanced data. IEEE Transactions on Cybernetics. 43(6): 1672-1687.

Castro, C. L., \& Braga, A. P. (2013). Novel cost-sensitive approach to improve the multilayer perceptron performance on imbalanced data. IEEE Transactions on Neural Networks and Learning Systems. 24(6): 888-899.

Cawley, G. C., \& Talbot, N. L. C. (2003). Efficient leave-one-out cross-validation of kernel Fisher discriminant classifier. Pattern Recognition. 36(12): 2585-2592.

Cooke, T. (2002). Two variations on Fisher's linear discriminant for pattern recognition. IEEE Transactions on Pattern Analysis and Machine Intelligence. 24(2): 268-273.

Duda, R. O., Hart, P. E., \& Stork, D. G. (2000). Pattern Classification. (2nd ed.). New York: John Wiley \& Sons, Inc.

Elozondo, D. (2006). The linear separability problem: Some testing methods. IEEE Transactions on Neural Networks. $17(2): 330-344$.

Escalera, S., Tax, D. M. J., Pujol, O., Radeva, P., \& Duin, R. P. W. (2008). Subclass problem-dependent design for error-correcting output codes. IEEE Transactions on Pattern Analysis and Machine Intelligence. 30(6): 1041-1054.

Fernandez, A., Garcia, S., Jesus, M. J., \& Herrera, F. (2008). A study of the behavior of linguistic fuzzy rule based classification systems in the framework of imbalanced data-sets. Fuzzy Sets and Systems. 159(18): 2378-2398.

Fukunaga, K., \& Hayes, R. R. (1989). Effects of sample size in classifier design. IEEE Transactions on Pattern Analysis and Machine Intelligence. 11(8): 873-885.

Galar, M., Fernández, A., Barrenechea, E., \&Herrera, F. (2013). cEUSBoost Enhancing ensembles for highly imbalanced data-sets by evolutionary undersampling. Pattern Recognition. 46(12): 3460-3471.

Galar, M., Fernandez, A., Barrenechea, E., Bustince, H., \& Herrera, F. (2012). A review on ensembles for the class imbalance problem bagging-, boosting-, and hybrid-based approaches. IEEE Transactions on Systems, Man, and Cybernetics-Part C: Applications and Reviews. 42(4): 463-484.

Gao, D. Q., Ding, J. \& Zhu, C. M. (2014). Integrated Fisher linear discriminants: An empirical study. Pattern Recognition. 47(2): 789-805.

Gormann, R. P., \& Sejnowski, T. J. (1988). Analysis of hidden units in a layered network trained to classify sonar targets. Neural Networks. 1(1): 75-89.

Hand, D. J., \& Till, R. J. (2001). A simple generalization of the area under the ROC curve for multiple class classification problems. Machine Learning 45(2): 171-186.

Hoyle, D. C. (2011). Accuracy of pseudo-inverse covariance learning-A random matrix theory analysis. IEEE Transactions on Pattern Analysis and Machine Intelligence. 33(8): 1470-1481.

Huang, J., \& Ling, C. X., (2005). Using AUC and accuracy in evaluating learning algorithms. IEEE Transactions on Knowledge and Data Engineering. 17(3): 299-310.

Jayadeva, Khemchandani, R., \& Chandra, S. (2007). Twin support vector machines for pattern classification. IEEE Transactions on Pattern Analysis and Machine Intelligence. 29(5): 905-910.

Kim, J. S., \& Scott, C. D. (2010). L2 kernel classification. IEEE Transactions on Pattern Analysis and Machine Intelligence. 32(10): $1822-1831$.

Koford, J. S., \& Groner, G. F. (1966). The use of an adaptive threshold element to design a linear optimal pattern classifier. IEEE Transactions on Information Theory. 12(1): 42-50.

Li, N., Tsang, I. W., \& Zhou, Z. H. (2013). Efficient optimization of performance measures by classifier adaptation. IEEE Transactions on Pattern Analysis and Machine Intelligence. 35(6): 1370-1382.

Liu, B., Hao, Z. F., \& Tsang, E. C. C. (2008). Nesting one-against-one algorithm based on SVMs for pattern classification. IEEE Transactions on Neural Networks. 19(12): 2044-2052.

Maldonado, S., \& Lopez, J. (2014). Imbalanced data classification using second-order cone programming support vector machines. Pattern Recognition. 47(5): 2070-2079.

Nicolas, G. P., Javier, P. R., \& Aida de, H. G. (2012). OligoIS: Scalable instance selection for class-imbalanced data sets. IEEE Transactions on Cybernetics. 43(1): 332-346.

Raudys, S., \& Duin, R. (1998). Expected classification error of the Fisher linear classifier with pseudo-inverse covariance matrix, Pattern Recognition Letter. 19(5-6): 385-392.

Roy, A., Govil, S., \& Miranda, R. (1995). An algorithm to generate radial basis function (RBF)-like nets for classification problems. 
Neural Networks. 8(2): 179-201.

Rozza, A., Lombardi, G., Casiraghi, E., \& Campadelli, P. (2012). Novel Fisher discriminant classifiers. Pattern Recognition. 45(10): $3725-3737$.

Shen, C. H., \& Li, H. X. (2010). On the dual formulation of boosting algorithms. IEEE Transactions on Pattern Analysis and Machine Intelligence. 32(12): 2216-2231.

Sheskin, D. J. (2006). Handbook of Parametric and Nonparametric Statistical Procedures. (2nd ed.). London: Chapman \& Hall/CRC.

Suykens, J. A. K., Gestel, T. V., Brabanter, J. D., Moor, B. D., \& Vandewalle, J. (2002). Least Squares Support Vector Machines. Singapore: World Scientific Publishing.

Tang, Y., Zhang, Y. Q., Chawla, N. V., \& Krasser, S. (2009). SVMs modeling for highly unbalanced classification. IEEE Transactions on Systems, Man, and Cybernetics-Part B, Cybernetics. 39(1): 281-289.

Tapson, J. \& Schaik, A. van. (2013). Learning the pseudoinverse solution to network weights. Neural Networks. 45: 94-100.

Toh, K. A., Tran, Q. L., \& Srinivasan, D. (2004). Benchmarking a reduced multivariate polynomial pattern classifier. IEEE Transactions on Pattern Analysis and Machine Intelligence. 26(6): 740-755.

Wang, S., \& Yao, X. (2013). Relationships between diversity of classification ensembles and single-class performance measures. IEEE Transactions on Knowledge and Data Engineering. 25(1): 206-219. 


\section{Table captions:}

Table I, Solutions of PILDs and FLDs for three 2-dimensional synthetic binary-class datasets.

Table II, Five empirical thresholds related to distributed regions for a binary-class problem $\left\{\omega_{1}, \omega_{2}\right\}$ (Gao, Ding, \& Zhu, 2014).

Table III, The sizes, deviations and thresholds for an 1-dimensional binary-class dataset: $\boldsymbol{X}^{(1)}=\{-2,2,3\} ; \boldsymbol{X}^{(2)}=\{4,4.25$, $4.75,7\}$.

Table $I V$, The learning results of the iterative PILDs with all 5 iteration stages and 4 decomposition operations for the Adult dataset.

Table $V$, Numerical characteristics of 24 benchmark datasets.

Table VI, The OCAs (\%) of iterative PILDs with different thresholds for 24 benchmark test datasets.

Table VII, The paired rank sums $\left\{R^{+}, R^{-}\right\}_{q r}$ of signed-rank Wilcoxon test by $\left\{\right.$ PILDs_ $\theta_{q}$, PILDs_ $\left.\theta_{r}\right\}$ based on OCAs for 24 benchmark test datasets.

Table VIII, Accuracies and runtimes of iterative PILDs and FLDs (Gao, Ding, \& Zhu, 2014) with optimal thresholds for 24 benchmark datasets.

Appendix A

Table $I X$, Accuracies, iterative epochs and runtimes of iterative PILDs with different thresholds for 24 benchmark training datasets.

Appendix $B$

Table $X$, The paired rank sums $\left\{R^{+}, R^{-}\right\}_{q r}$ of signed-rank Wilcoxon test by $\left\{\right.$ PILDs_ $\theta_{q}$, PILDs_ $\left.\theta_{r}\right\}$ based on OCAs for 24 benchmark training datasets. 


\section{Figure captions:}

Fig. 1. Re-allocation of the desired output of a mislabeled negative sample. $\boldsymbol{w}$ : Weight vectors; $\oplus$ : Mean vectors; $\boldsymbol{\pi}$. Decision lines.

Fig. 2. Change of overall error rates, optimal threshold's indices and min and max algebraic distances by an iterative PILD for the Sonar dataset.

Fig. 3. Overall error rates, optimal threshold indices, and min and max algebraic distances by an iterative PILD for the Adult training dataset. 


\section{Table I, Solutions of PILDs and FLDs for 3 two-class datasets.}

Table I, Solutions of PILDs and FLDs for three synthetic 2-dimensional binary-class datasets.

\begin{tabular}{|c|c|c|c|c|c|c|}
\hline Dataset & Sample distribution & Solution Item & $\begin{array}{c}\text { PILD } \\
d_{p} \in\left\{N / N_{1},-N / N_{2}\right\}\end{array}$ & $\begin{array}{c}\text { PILD } \\
d_{p} \in\{1,-1\}\end{array}$ & $\begin{array}{c}\text { FLD } \\
\theta_{1}=-\mu_{w}\end{array}$ & $\begin{array}{c}\text { FLD } \\
\theta_{2}=-\left(\mu_{1}+\mu_{2}\right) / 2\end{array}$ \\
\hline 1 & $\begin{array}{l}\omega_{1}:(\alpha, 0)^{T},(0,1)^{T},(0,-1)^{T} ; \\
\omega_{2}:(1,0)^{T} .\end{array}$ & $\begin{array}{c}\text { Decision line } \\
\text { Active region of } \alpha\end{array}$ & $\begin{array}{c}4 x_{1}-(\alpha+1)=0 \\
-1<\alpha<1 / 3\end{array}$ & $\begin{array}{c}2(\alpha-3) x_{1}+\alpha^{2}+3=0 \\
-3<\alpha<1\end{array}$ & $\begin{array}{c}4 x_{1}-(\alpha+1)=0 \\
(-1<\alpha<1 / 3) \cap(\alpha \neq 0)\end{array}$ & $\begin{array}{c}6 x_{1}-(\alpha+3)=0 \\
(-3<\alpha<3 / 5) \cap(\alpha \neq 0)\end{array}$ \\
\hline 2 & $\begin{array}{l}\omega_{1}:(\alpha, \alpha)^{T},(0,1)^{T},(1,0)^{T} ; \\
\omega_{2}:(1,1)^{T} .\end{array}$ & $\begin{array}{l}\text { Decision line } \\
\text { Active region of } \alpha\end{array}$ & $\begin{array}{c}2 x_{1}+2 x_{2}-(\alpha+2)=0 \\
0<\alpha<2 / 3\end{array}$ & $\begin{array}{c}(\alpha-2) x_{1}+(\alpha-2) x_{2}+\alpha^{2}-2 \alpha+3=0 \\
-1<\alpha<1\end{array}$ & $\begin{array}{c}2 x_{1}+2 x_{2}-(\alpha+2)=0 \\
(0<\alpha<2 / 3) \cap(\alpha \neq 1 / 2)\end{array}$ & $\begin{array}{c}3 x_{1}+3 x_{2}-(\alpha+4)=0 \\
(-1<\alpha<4 / 5) \cap(\alpha \neq 1 / 2)\end{array}$ \\
\hline 3 & $\begin{array}{l}\omega_{1}:(1,2)^{T},(2.0)^{T} \\
\omega_{2}:(3.1)^{T},(2.3)^{T} .\end{array}$ & Decision line & $-4 x_{1}-2 x_{2}+11=0$ & $-4 x_{1}-2 x_{2}+11=0$ & $\begin{array}{l}\text { Singular } \boldsymbol{S}_{w} \\
\text { No available }\end{array}$ & $\begin{array}{l}\text { Singular } \boldsymbol{S}_{w} \\
\text { No available }\end{array}$ \\
\hline
\end{tabular}




\section{Table II, 5 empirical thresholds related to distributed regions}

Table II, Five empirical thresholds related to distribution regions for a binary-class problem $\left\{\omega_{1}, \omega_{2}\right\}$ (Gao, Ding, \& Zhu, 2014).

\begin{tabular}{|c|c|c|c|c|c|}
\hline Threshold & $\theta_{7}$ & $\theta_{8}$ & $\theta_{9}$ & $\theta_{10}$ & $\theta_{11}$ \\
\hline $\begin{array}{l}\text { Regional } \\
\text { variables }\end{array}$ & $\sigma_{w}^{(1)}, \sigma_{w}^{(2)}$ & $\delta_{w}^{(1)}, \delta_{w}^{(2)}$ & $\delta_{\boldsymbol{w}_{-} i n}^{(1)}, \quad \delta_{\boldsymbol{w}_{-} i n}^{(2)}$ & $\bar{\delta}_{\boldsymbol{w}}^{(1)}, \bar{\delta}_{\boldsymbol{w}}^{(2)}$ & $\bar{\delta}_{\boldsymbol{w}_{-} i n}^{(1)}, \bar{\delta}_{\boldsymbol{w}_{-} i n}^{(2)}$ \\
\hline $\begin{array}{l}\text { Computing } \\
\text { formula }\end{array}$ & $-\frac{\sigma_{w}^{(2)} \mu_{w}^{(1)}+\sigma_{w}^{(1)} \mu_{w}^{(2)}}{\sigma_{w}^{(1)}+\sigma_{w}^{(2)}}$ & $-\frac{\delta_{w}^{(2)} \mu_{w}^{(1)}+\delta_{w}^{(1)} \mu_{w}^{(2)}}{\delta_{w}^{(1)}+\delta_{w}^{(2)}}$ & $-\frac{\delta_{\boldsymbol{w}_{-} i n}^{(2)} \mu_{\boldsymbol{w}^{\prime}}^{(1)}+\delta_{\mathbf{w}_{-} i n}^{(1)} \mu_{\boldsymbol{w}}^{(2)}}{\delta_{\boldsymbol{w}_{-} i n}^{(1)}+\delta_{\boldsymbol{w}_{-} i n}^{(2)}}$ & $-\frac{\bar{\delta}_{w}^{(2)} \mu_{w}^{(1)}+\bar{\delta}_{w}^{(1)} \mu_{w}^{(2)}}{\bar{\delta}_{w}^{(1)}+\bar{\delta}_{w}^{(2)}}$ & $-\frac{\bar{\delta}_{\mathbf{w}_{-} i n}^{(2)} \mu_{\boldsymbol{w}}^{(1)}+\bar{\delta}_{\boldsymbol{w}_{-} i n}^{(1)} \mu_{\mathbf{w}}^{(2)}}{\bar{\delta}_{\mathbf{w}_{-} i n}^{(1)}+\bar{\delta}_{\mathbf{w}_{-} i n}^{(2)}}$ \\
\hline
\end{tabular}


Table III, Sizes, deviations \& thresholds for a 1D 2-class data.

Table III, The sizes, deviations and thresholds by a PILD for an 1-dimensional binary-class dataset: $\boldsymbol{X}^{(1)}=\{-2,2,3\} ; \boldsymbol{X}^{(2)}=\{4,4.25,4.75,7\}$.

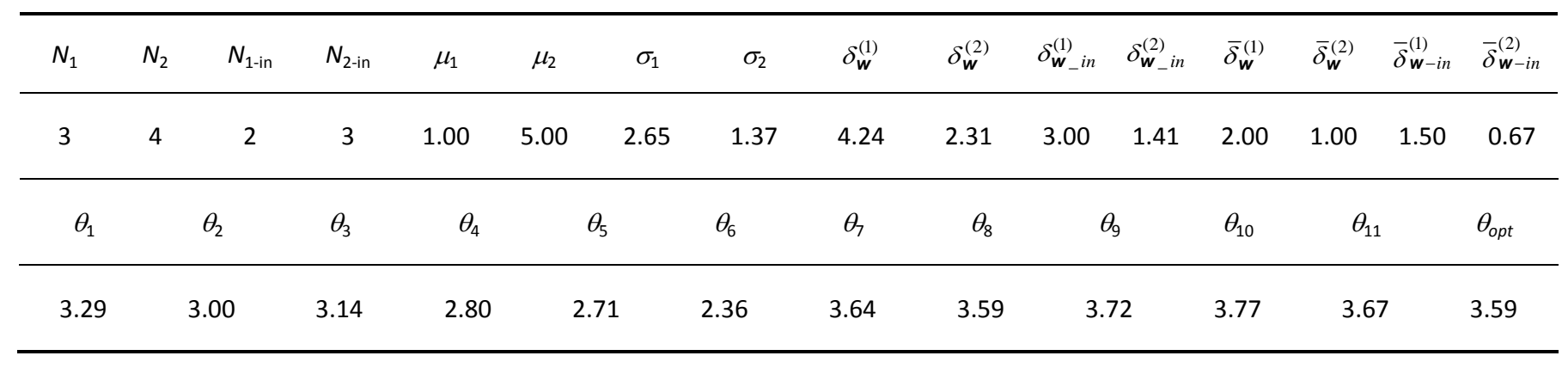


Table IV, Results of PILDs with 5 iteration stages for Adult.

Table IV, The learning results of the iterative PILDs with all 5 iteration stages and 4 decomposition operations for the Adult dataset.

\begin{tabular}{|c|c|c|c|c|c|c|c|c|}
\hline \multirow{2}{*}{$\begin{array}{l}\text { Iteration } \\
\text { stage }\end{array}$} & \multicolumn{3}{|c|}{ No. learning samples } & \multirow{2}{*}{$\begin{array}{l}\text { \#Iteration } \\
\text { epochs }\end{array}$} & \multicolumn{2}{|c|}{ Mislabeled samples } & \multirow{2}{*}{$\begin{array}{c}\text { Mean interval } \\
{\left[\mu_{1}, \mu_{2}\right]}\end{array}$} & \multirow{2}{*}{$\begin{array}{c}\text { Algebraic Euclid } \\
\text { distances }\left(d_{\min }, d_{\max }\right)\end{array}$} \\
\hline & Sum & Minority class & Majority class & & Number & Error rate $(\%)$ & & \\
\hline $1^{\mathrm{st}}$ & 30162 & 7508 & 22654 & 32 & 5026 & 16.66 & $0.199,-0.066$ & $(4.14,-0.51) \times 10^{-4}$ \\
\hline $2^{\text {nd }}$ & 15438 & 4832 & 10606 & 11 & 4969 & 16.47 & $0.038,0.014$ & $(2.51,-5.69) \times 10^{-6}$ \\
\hline $3^{\mathrm{rd}}$ & 4747 & 1532 & 3215 & 5 & 4981 & 16.51 & $0.013,0.012$ & $(1.17,-5.88) \times 10^{-7}$ \\
\hline $4^{\text {th }}$ & 339 & 121 & 218 & 7 & 5092 & 16.88 & $(1.08,0.93) \times 10^{-3}$ & $(1.07,-3.00) \times 10^{+8}$ \\
\hline $5^{\text {th }}$ & 80 & 31 & 49 & 5 & 6308 & 20.91 & $(6.61,1.31) \times 10^{6}$ & 183.90. - 288.39 \\
\hline
\end{tabular}


Table $V$, Numerical characteristics of 24 benchmark datasets.

\begin{tabular}{|c|c|c|c|c|c|c|c|}
\hline \multirow{2}{*}{ Dataset } & \multicolumn{2}{|c|}{ Training samples } & \multirow{2}{*}{$\begin{array}{l}\text { No. test } \\
\text { samples }\end{array}$} & \multicolumn{2}{|c|}{ Attribute } & \multirow{2}{*}{$\begin{array}{c}\text { No. } \\
\text { classes }\end{array}$} & \multirow{2}{*}{ Reference $^{* *}$} \\
\hline & No. & $I R_{\max }$ & & Type $^{*}$ & No. & & \\
\hline Image segment PCA7 & 210 & 1 & 2100 & $\mathrm{I}, \mathrm{R}$ & 7 & 7 & (a) \\
\hline Optdigit & 3823 & 1.03 & 1797 & I & 64 & 10 & (a) \\
\hline Pendigit & 7494 & 1.08 & 3498 & I & 16 & 10 & (a) \\
\hline Sonar & 104 & 1.12 & 104 & $\mathrm{R}$ & 60 & 2 & (a) \\
\hline Letter & 16000 & 1.13 & 4000 & 1 & 16 & 26 & (a) \\
\hline Heart disease & 180 & 1.25 & 90 & $B, I, N, R$ & 13 & 2 & (a) \\
\hline Liver disorder & 230 & 1.37 & 115 & $\mathrm{I}, \mathrm{R}$ & 6 & 2 & (a) \\
\hline 2D Vowel & 338 & 1.74 & 333 & 1 & 2 & 10 & (c) \\
\hline Ionosphere & 234 & 1.79 & 117 & $\mathrm{R}$ & 33 & 2 & (a) \\
\hline Splice- $b$ & 2117 & 2.16 & 1058 & N/B & 120 & 3 & (a) \\
\hline German credit & 667 & 2.34 & 333 & $\mathrm{I}, \mathrm{N}$ & 24 & 2 & (a) \\
\hline Satellite image & 4435 & 2.58 & 2000 & 1 & 36 & 6 & (a) \\
\hline Adult & 30162 & 3.02 & 15060 & $\mathrm{I}, \mathrm{N}$ & 14 & 2 & (a) \\
\hline Balance & 417 & 5.82 & 208 & $\mathrm{R}$ & 4 & 3 & (a) \\
\hline Glass & 143 & 8.50 & 71 & $\mathrm{I}, \mathrm{R}$ & 9 & 6 & (a) \\
\hline Abalone19v18_5CV & 584.8 & 16.73 & 146.2 & $\mathrm{~N}, \mathrm{R}$ & 8 & 2 & (b) \\
\hline Thyroid & 3772 & 37.51 & 3428 & $B, R$ & 21 & 3 & (a) \\
\hline Lymphography_5CV & 118.4 & 65 & 29.8 & $B, R$ & 18 & 4 & (b) \\
\hline Yeast & 989 & 103 & 495 & $\mathrm{R}$ & 8 & 10 & (a) \\
\hline Ecoli_5CV & 268.8 & 115 & 68 & $\mathrm{R}$ & 7 & 8 & (b) \\
\hline Abalone19 & 2782 & 131.48 & 1392 & $N, R$ & 8 & 2 & (b) \\
\hline Abalon19_5CV & 3339.2 & 132.56 & 834.8 & $N, R$ & 8 & 2 & (b) \\
\hline Pageblocks_5CV & 438.4 & 197 & 109.6 & $\mathrm{R}$ & 10 & 5 & (b) \\
\hline Shuttle & 43500 & 5684.67 & 14500 & I & 9 & 7 & (a) \\
\hline
\end{tabular}

*: $B$ - Binary; I-Integer; $R$ - Real number; $N$ - Nominal.

**: (a)-(Asuncion \& Newman, 2010); (b)-(Alcala-Fdez et al., 2011); (c)-(Roy, Govil, \& Miranda, 1995). 
Table VI, OCAs of iterative PILDs for 24 benchmark test data.

Table VI, The OCAs (\%) of iterative PILDs with different thresholds for 24 benchmark test datasets.

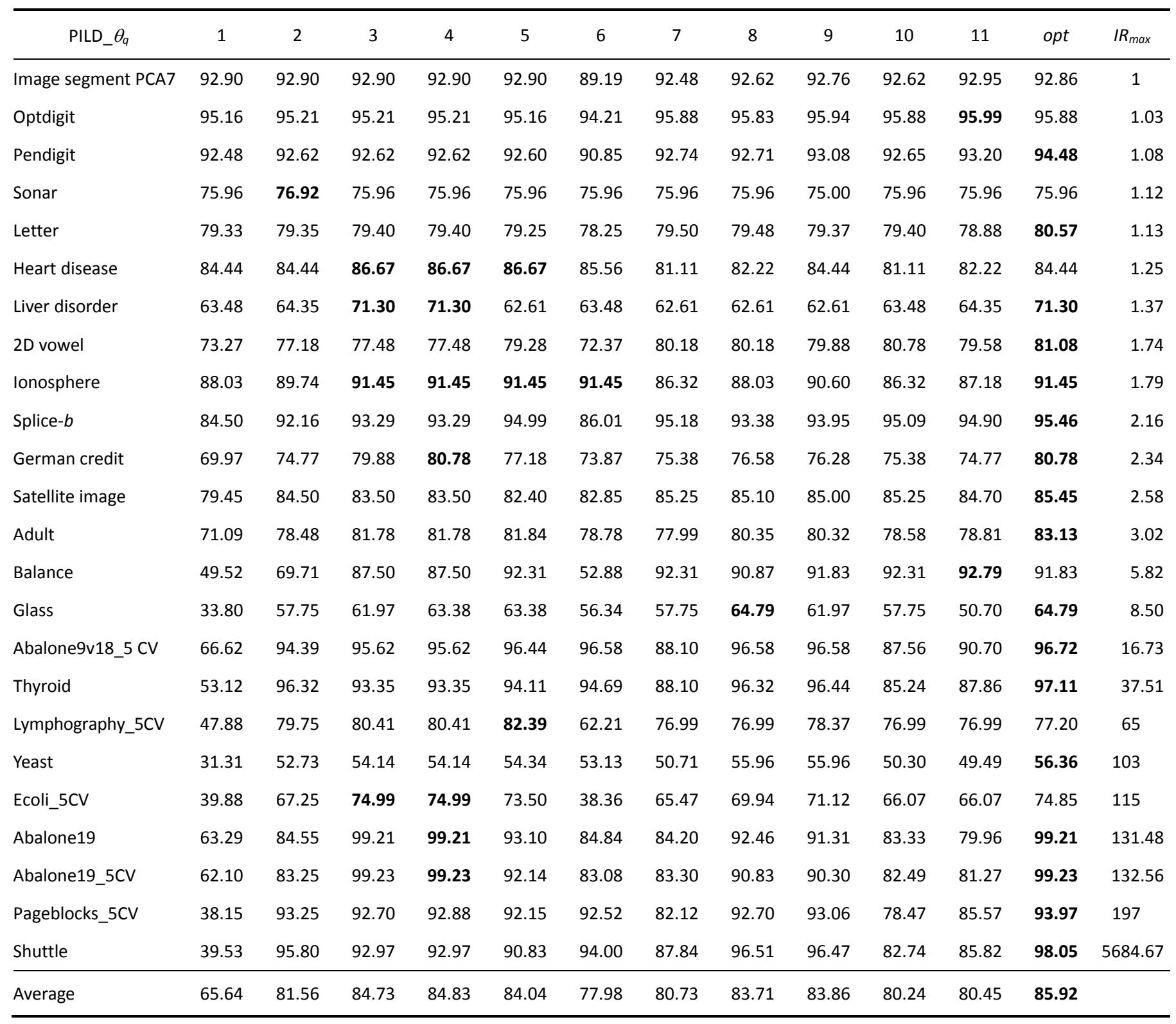


Table VII, Wilcoxon paired rank sums for 24 benchmark test data.

Table VII, The paired rank sums $\left\{R^{+}, R^{-}\right\}_{q r}$ of signed-rank Wilcoxon test by $\left\{\right.$ PILDs_ $\theta_{q}$, PILDs_ $\left.\theta_{r}\right\}$ based on OCAs for 24 benchmark test datasets.

\begin{tabular}{|c|c|c|c|c|c|c|c|c|c|c|c|}
\hline$q^{r}$ & 1 & 2 & 3 & 4 & 5 & 6 & 7 & 8 & 9 & 10 & 11 \\
\hline opt & $\{252,1\}$ & $\{257,-19\}$ & $\{159,-31\}$ & $\{141,-30\}$ & $\{216,-37\}$ & $\{251,-2\}$ & $\{248,-5\}$ & $\{253,0\}$ & $\{241.5,-10.5\}$ & $\{247,-6\}$ & $\{267,-9\}$ \\
\hline 11 & $\{262,-14\}$ & $\{63,-190\}$ & $\{41,-235\}$ & $\{40,-236\}$ & $\{39,-237\}$ & $\{145,-131\}$ & $\{118,-135\}$ & $\{37,-194\}$ & $\{43,-257\}$ & $\{127.5,-103.5\}$ & \\
\hline 10 & $\{239,-14\}$ & $\{80,-196\}$ & $\{32,-221\}$ & $\{30,-223\}$ & $\{33,-220\}$ & $\{140,-113\}$ & $\{39.5,-80.5\}$ & $\{36,-195\}$ & $\{47,-253\}$ & & \\
\hline 9 & $\{263,-13\}$ & $\{232,-44\}$ & $\{117,-159\}$ & $\{122,-178\}\{1$ & $109.5,-166.5\}$ & $\{261,-15\}$ & $\{240,-36\}$ & $\{140,-91\}$ & & & \\
\hline 8 & $\{239,-14\}$ & $\{208,-68\}$ & $\{98,-155\}$ & $\{104,-172\}$ & $\{109,-144\}$ & $\{226,-27\}$ & $\{181,-29\}$ & & & & \\
\hline 7 & $\{255,-21\}$ & $\{84,-192\}$ & $\{38,-238\}$ & $\{36,-240\}$ & $\{25,-206\}$ & $\{160,-116\}$ & & & & & \\
\hline 6 & $\{223,-30\}$ & $\{53,-247\}$ & $\{18,-235\}$ & $\{18,-235\}$ & $\{27,-226\}$ & & & & & & \\
\hline 5 & $\{227,-4\}$ & $\{212.5,-63.5\}$ & $\{89,-121\}$ & $\{75,-115\}$ & & & & & & & \\
\hline 4 & $\{253,0\}$ & $\{192,-39\}$ & $\{6,0\}$ & & & & & & & & \\
\hline 3 & $\{253,0\}$ & $\{192,-39\}$ & & & & & & & & & \\
\hline 2 & $\{253,0\}$ & & & & & & & & & & \\
\hline
\end{tabular}


Table VIII, OCAs \& runtimes of PILDs \& FLDs for 24 data.

Table VIII, Accuracies and runtimes of iterative PILDs and FLDs (Gao, Ding, \& Zhu, 2014) with optimal thresholds for 24 benchmark datasets.

\begin{tabular}{|c|c|c|c|c|c|c|c|c|c|c|c|c|c|}
\hline \multirow{2}{*}{ Dataset } & \multicolumn{2}{|c|}{ Training OCA (\%) } & \multicolumn{2}{|c|}{ Test OCA (\%) } & \multicolumn{2}{|c|}{ Runtime (s) } & \multirow{2}{*}{ Dataset } & \multicolumn{2}{|c|}{ Training OCA (\%) } & \multicolumn{2}{|c|}{ Test OCA (\%) } & \multicolumn{2}{|c|}{ Runtime (s) } \\
\hline & $\mathrm{PILD}_{o p t}$ & $\mathrm{FLD}_{12}$ & $\mathrm{PILD}_{\text {opt }}$ & $\mathrm{FLD}_{12}$ & $\mathrm{PILD}_{\text {opt }}$ & $\mathrm{FLD}_{12}$ & & $\mathrm{PILD}_{\text {opt }}$ & $\mathrm{FLD}_{12}$ & $\mathrm{PILD}_{\text {opt }}$ & $\mathrm{FLD}_{12}$ & $\mathrm{PILD}_{o p t}$ & $\mathrm{FLD}_{12}$ \\
\hline Optdiagit & 99.63 & 99.42 & 95.88 & 96.05 & 15.81 & 6.86 & Balance & 90.89 & 90.89 & 91.83 & 91.83 & 0.34 & 0.12 \\
\hline Sonar & 100.00 & 92.31 & 75.96 & 75.96 & 0.19 & 1.05 & $\begin{array}{l}\text { Abalone9v18_ } \\
5 \mathrm{CV}\end{array}$ & 96.72 & 96.24 & 96.72 & 96.44 & 1.57 & 0.53 \\
\hline Letter & 84.49 & 86.20 & 80.57 & 82.30 & 246.36 & 59.33 & Thyroid & 98.14 & 98.41 & 97.11 & 97.70 & 6.21 & 2.36 \\
\hline Heart disease & 88.33 & 87.78 & 84.44 & 86.67 & 0.16 & 0.28 & $\begin{array}{l}\text { Lymphography_ } \\
5 \mathrm{CV}\end{array}$ & 92.06 & 79.69 & 77.20 & 71.86 & 0.70 & 0.89 \\
\hline lonosphere & 90.60 & 92.74 & 91.45 & 94.02 & 0.12 & 0.36 & Abalone19 & 99.25 & 90.69 & 99.21 & 93.10 & 1.39 & 0.66 \\
\hline Splice- $b$ & 96.93 & 97.64 & 95.46 & 94.52 & 3.42 & 3.06 & Abalone19_5CV & 99.23 & 91.89 & 99.23 & 92.14 & 7.13 & 0.81 \\
\hline German credit & 78.26 & 75.41 & 80.78 & 77.18 & 0.20 & 0.44 & $\begin{array}{l}\text { Pageblocks__ } \\
5 \mathrm{CV}\end{array}$ & 97.63 & 95.80 & 93.97 & 92.69 & 2.18 & 1.45 \\
\hline \multirow[t]{2}{*}{ Satellite image } & 87.73 & 88.50 & 85.45 & 85.50 & 8.80 & 3.89 & Shuttle & 98.11 & 98.15 & 98.05 & 98.15 & 689.19 & 105.84 \\
\hline & & & & & & & Average & 89.88 & 86.61 & 85.92 & 83.60 & 50.68 & 9.54 \\
\hline
\end{tabular}


Appendix A

Table IX, Accuracies, epochs and runtimes of iterative PILDs with different thresholds for 24 benchmark training datasets.

\begin{tabular}{|c|c|c|c|c|c|c|c|c|c|c|c|c|c|c|}
\hline PILD_ $\theta_{q}$ & \multicolumn{12}{|c|}{ OCAs (\%) } & \multicolumn{2}{|c|}{ Iterative learning } \\
\hline Optdigit & 98.59 & 98.56 & 98.56 & 98.56 & 98.54 & 98.09 & 98.64 & 98.61 & 98.64 & 98.59 & 98.64 & 99.63 & 628 & 15.81 \\
\hline Pendigit & 97.00 & 97.00 & 97.02 & 97.02 & 97.06 & 95.78 & 97.08 & 97.04 & 97.16 & 97.02 & 97.06 & 98.68 & 664 & 24.59 \\
\hline Letter & 81.23 & 81.29 & 81.36 & 81.36 & 81.31 & 80.14 & 81.33 & 81.27 & 81.41 & 81.19 & 81.46 & 84.49 & 7793 & 246.36 \\
\hline Heart disease & 86.67 & 86.67 & 87.78 & 87.78 & 87.78 & 87.22 & 85.56 & 86.11 & 87.22 & 85.00 & 86.11 & 88.33 & 8 & 0.16 \\
\hline Liver disorder & 65.22 & 66.09 & 70.00 & 70.00 & 66.09 & 66.52 & 66.09 & 66.09 & 66.09 & 66.52 & 66.96 & 71.30 & 15 & 0.11 \\
\hline Splice- $b$ & 85.26 & 93.43 & 94.99 & 94.99 & 96.41 & 87.25 & 96.46 & 95.51 & 95.89 & 96.50 & 96.27 & 96.93 & 31 & 3.42 \\
\hline German credit & 70.31 & 73.76 & 77.51 & 78.26 & 74.81 & 72.71 & 74.36 & 73.91 & 73.76 & 74.06 & 74.06 & 78.26 & 11 & 0.20 \\
\hline Satellite image & 82.16 & 86.72 & 86.85 & 86.85 & 85.12 & 84.40 & 86.90 & 87.03 & 87.19 & 86.67 & 86.47 & 87.73 & 211 & 8.80 \\
\hline Adult & 71.56 & 78.97 & 81.80 & 81.80 & 82.18 & 79.38 & 78.37 & 81.13 & 81.09 & 79.13 & 79.43 & 83.34 & 60 & 196.02 \\
\hline Balance & 53.00 & 72.42 & 89.21 & 89.21 & 89.93 & 55.16 & 90.89 & 89.21 & 89.21 & 90.89 & 90.89 & 90.89 & 61 & 0.34 \\
\hline Glass & 60.14 & 71.33 & 76.22 & 77.62 & 70.63 & 69.23 & 78.32 & 78.32 & 77.62 & 77.62 & 75.52 & 80.42 & 38 & 0.39 \\
\hline Abalone9v18_5 CV & 67.37 & 93.74 & 95.52 & 95.52 & 95.97 & 96.24 & 87.14 & 95.79 & 96.17 & 86.80 & 89.53 & 96.72 & 120 & 1.57 \\
\hline Abalone19_5CV & 62.20 & 83.06 & 99.23 & 99.23 & 91.89 & 82.97 & 83.17 & 90.75 & 90.15 & 82.52 & 81.40 & 99.23 & 175 & 7.13 \\
\hline Pageblocks_5CV & 44.25 & 96.44 & 94.44 & 96.40 & 93.94 & 94.57 & 83.90 & 96.49 & 96.81 & 80.29 & 87.45 & 97.63 & 184 & 2.18 \\
\hline Shuttle & 40.02 & 96.10 & 92.78 & 92.78 & 90.31 & 93.81 & 88.31 & 96.44 & 96.31 & 83.36 & 86.47 & 98.11 & 417 & 689.19 \\
\hline Average & 69.35 & 84.43 & 87.56 & 87.94 & 86.37 & 80.49 & 84.16 & 86.84 & 86.88 & 83.51 & 84.16 & 89.88 & 498.75 & 50.68 \\
\hline
\end{tabular}




\section{Table $\mathrm{X}$, Wilcoxon pair rank sums for 24 benchmark training data.}

Appendix B

Table $X$, The paired rank sums $\left\{R^{+}, R^{-}\right\}_{q r}$ of signed-rank Wilcoxon test by $\left\{\right.$ PILDs_ $\theta_{q}$, PILDs_ $\left.\theta_{r}\right\}$ based on OCAs for 24 benchmark training datasets.

\begin{tabular}{|c|c|c|c|c|c|c|c|c|c|c|c|}
\hline$q^{r}$ & 1 & 2 & 3 & 4 & 5 & 6 & 7 & 8 & 9 & 10 & 11 \\
\hline opt & $\{300,0\}$ & $\{300,0\}$ & $\{253,0\}$ & $\{210,0\}$ & $\{276,0\}$ & $\{300,0\}$ & $\{276,0\}$ & $\{300,0\}$ & $\{300,0\}$ & $\{276,0\}$ & $\{253,0\}$ \\
\hline 10 & $\{260,-16\}$ & $\{118,-182\}$ & $\{44,-232\}$ & $\{26,-227\}$ & $\{64,-212\}$ & $\{161,-115\}$ & $\{40.5,-212.5\}$ & $\{35,-218\}$ & $\{46.5,-229.5\}$ & & \\
\hline 7 & $\{241,-35\}$ & $\{40,-236\}$ & $\{22,-278\}$ & $\{18,-282\}$ & $\{30,-270\}$ & $\{106,-170\}$ & & & & & \\
\hline 6 & $\{283,-17\}$ & $\{119.5,-156.5\}$ & $\{63,-237\}$ & $\{49,-251\}$ & $\{70,-203\}$ & & & & & & \\
\hline 5 & $\{275,-1\}$ & $\{180.5,-72.5\}$ & $\{83,-170\}$ & $\{60,-193\}$ & & & & & & & \\
\hline 2 & $\{230,-1\}$ & & & & & & & & & & \\
\hline
\end{tabular}


Fig. 1. Re-allocating desired output of a mislabel. neg. sample.

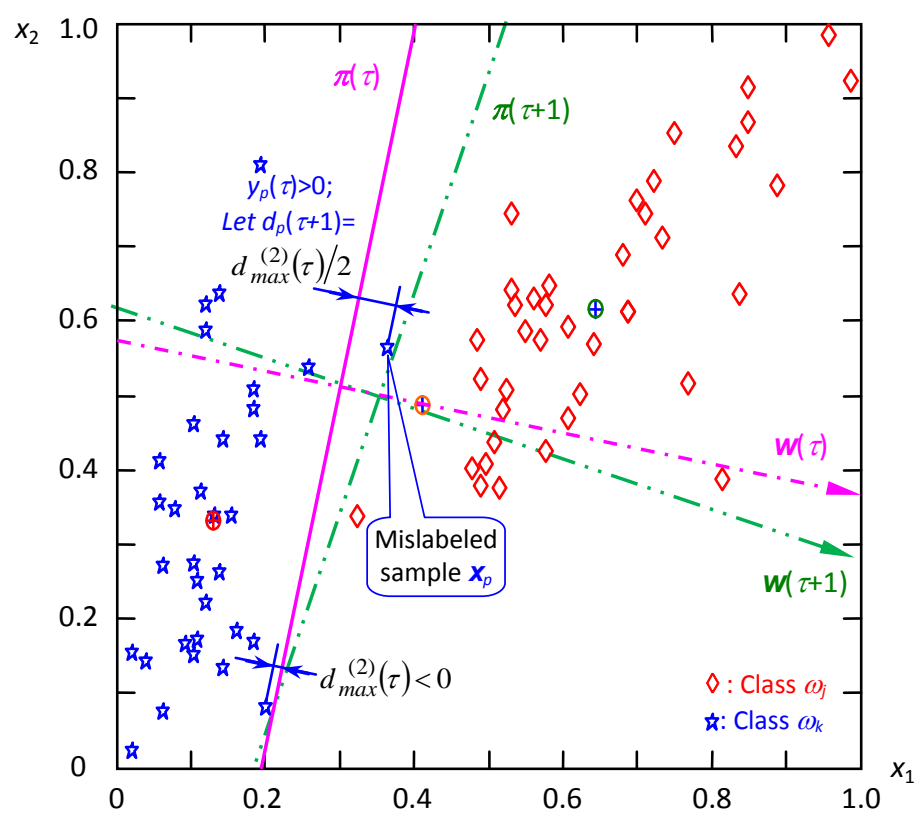

Fig. 1. Re-allocating the desired output of a mislabeled negative sample.

w: Weight vectors; $\oplus$ : Mean vectors; $\pi$. Decision lines. 
Fig. 2. OERs, thres. indices $\&$ alg. Distan. by a PILD for Sonar.

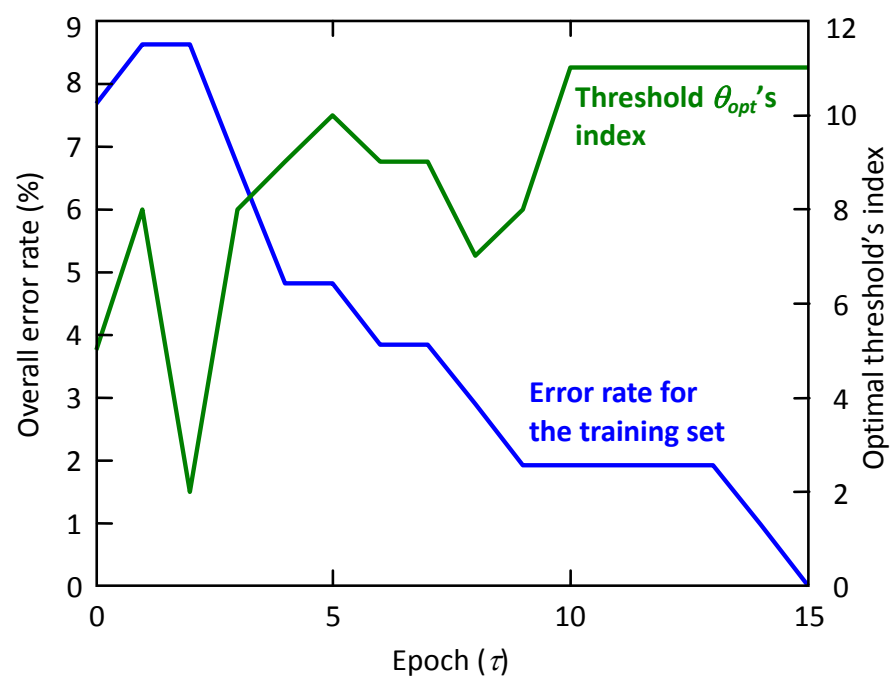

(a)

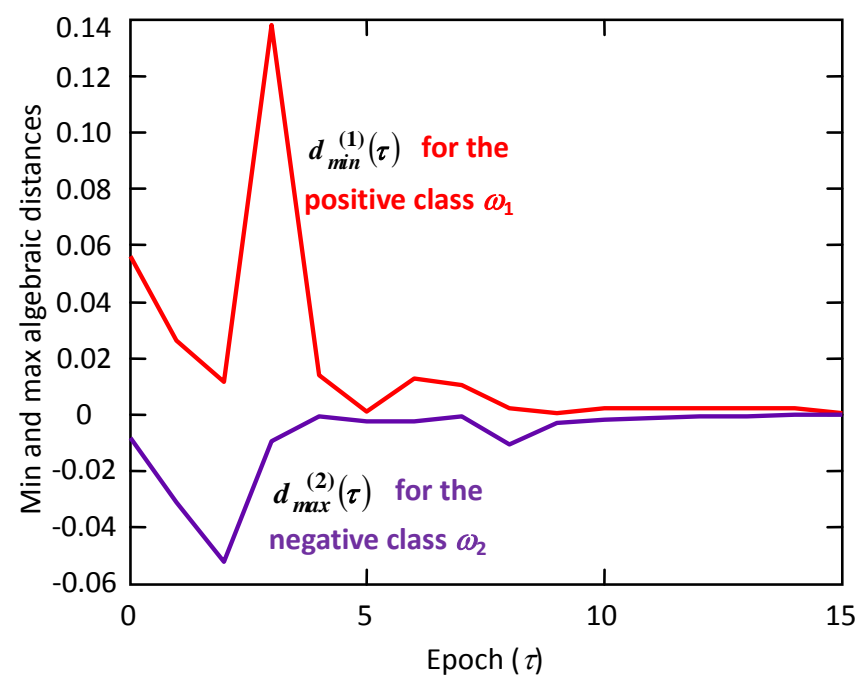

(b)

Fig. 2. Change of overall error rates, optimal threshold's indices and min and max algebraic distances by an iterative PILD for the Sonar dataset.

(a), Overall error rate and optimal threshold's indices. (b), Minimum and maximum algebraic Euclidean distances. 
Fig. 3. OERs, thres. indices \& Distances for Adult training data

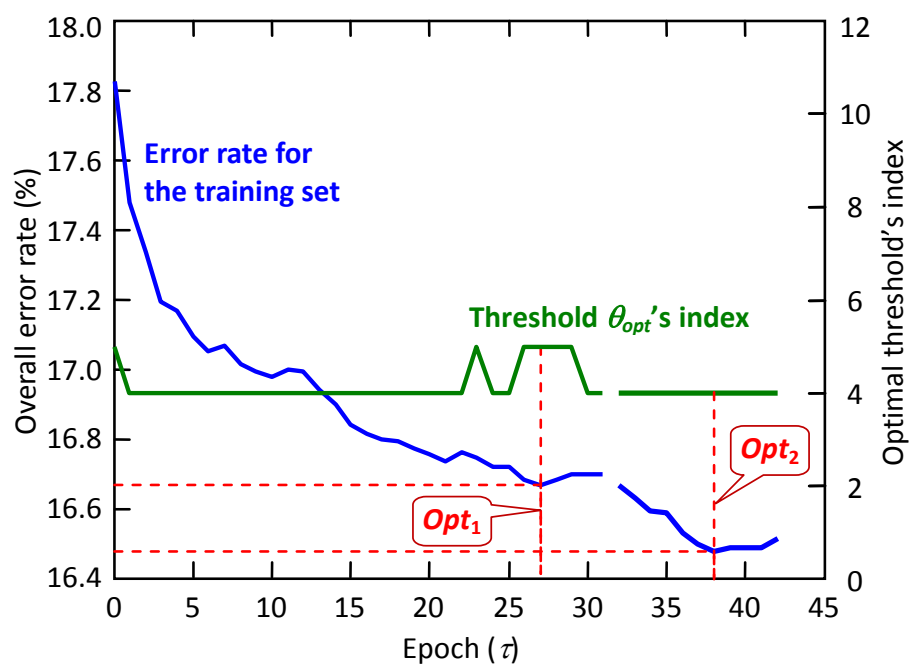

(a)

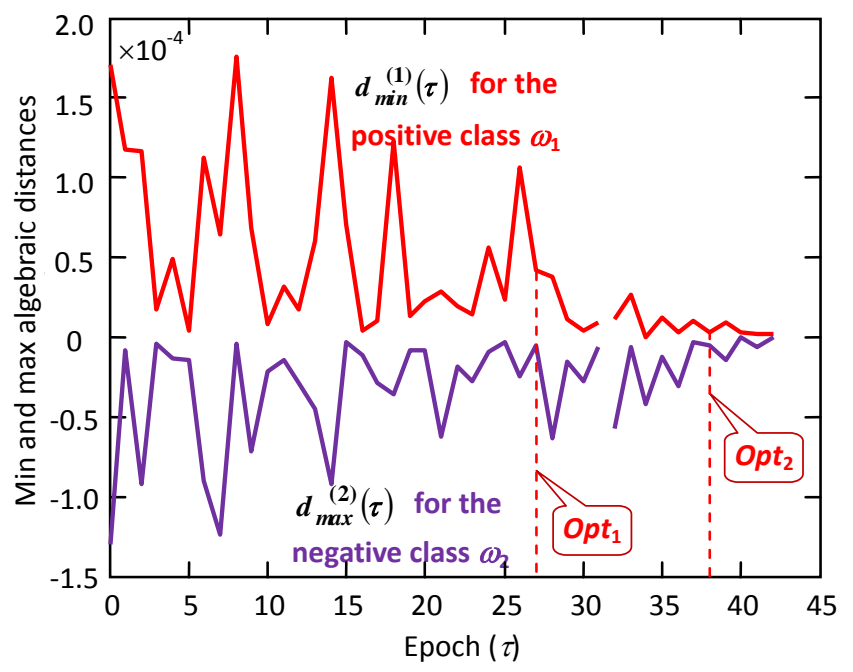

(b)

Fig. 3. Overall error rates, optimal threshold indices, and min and max algebraic distances by an iterative PILD for the Adult training dataset.

(a), Overall error rate and optimal threshold's index. (b), Minimum and maximum algebraic Euclidean distances. 